\title{
Isochrone fitting of Galactic globular clusters - II. NGC 6205 (M13)
}

\author{
George A. Gontcharov, ${ }^{1,2 \star}$ Maxim Yu. Khovritchev ${ }^{3,4}$ and Aleksandr V. Mosenkov ${ }^{3}$ \\ ${ }^{1}$ Department for Management of Science and Technology Development, Ton Duc Thang University, Ho Chi Minh City, Vietnam \\ ${ }^{2}$ Faculty of Applied Sciences, Ton Duc Thang University, Ho Chi Minh City, Vietnam \\ ${ }^{3}$ Central Astronomical Observatory, Russian Academy of Sciences, 65/1 Pulkovskoye chaussee, St. Petersburg 196140, Russia \\ ${ }^{4}$ St. Petersburg State University, $7 / 9$ Universitetskaya nab., St. Petersburg 199034, Russia
}

Accepted 2020 June 10. Received 2020 June 9; in original form 2020 March 16

\begin{abstract}
We present new isochrone fits to colour-magnitude diagrams of the Galactic globular cluster NGC 6205 (M13). We utilise 34 photometric bands from the ultraviolet to midinfrared by use of data from the HST, Gaia DR2, SDSS, unWISE, Pan-STARRS DR1, and other photometric sources. In our isochrone fitting we use the PARSEC, MIST, DSEP, BaSTI, and IAC-BaSTI theoretical models and isochrones, both for the solarscaled and $\mathrm{He}-\alpha$-enhanced abundances, with a metallicity of about $[\mathrm{Fe} / \mathrm{H}]=-1.58$ adopted from the literature. The colour-magnitude diagrams, obtained with pairs of filters from different datasets but of similar effective wavelengths, show some colour offsets up to 0.04 mag between the fiducial sequences and isochrones. We attribute these offsets to systematic differences of the datasets. Some intrinsic systematic differences of the models/isochrones remain in our results: the derived distances and ages are different for the ultraviolet, optical and infrared photometry used, while the derived ages are different for the different models/isochrones, e.g. in the optical range from $12.3 \pm 0.7$ Gyr for He- $\alpha$-enhanced DSEP to 14.4 \pm 0.7 Gyr for MIST. Despite the presence of multiple stellar populations, we obtain convergent estimates for the dominant population: best-fitting distance $7.4 \pm 0.2 \mathrm{kpc}$, true distance modulus $14.35 \pm 0.06 \mathrm{mag}$, parallax $0.135 \pm 0.004$ mas, extinction $A_{\mathrm{V}}=0.12 \pm 0.02$, and reddening $E(B-V)=0.04 \pm 0.01$. These estimates agree with other recent estimates, however, the extinction and reddening are twice as high as generally accepted. The derived empirical extinction law agrees with the Cardelli-Clayton-Mathis extinction law with the best-fitting $R_{\mathrm{V}}=3.1_{-1.1}^{+1.6}$.
\end{abstract}

Key words: Hertzsprung-Russell and colour-magnitude diagrams - dust, extinction - globular clusters: general - globular clusters: individual: NGC 6205 (M13)

\section{INTRODUCTION}

Multiple stellar populations have been found in all or almost all Galactic globular clusters (GCs) (Monelli et al. 2013). In particular, such populations can be seen using appropriate filters, colours, and colour combinations, which are sensitive to them. This makes it difficult to obtain accurate cluster properties from isochrone fits to colour-magnitude diagrams (CMDs). Using a CMD, one must either indicate that a difference between the positions of the populations in that CMD is negligible or that a dominant population exists and can be well separated in that CMD from other populations. If this is the case for several CMDs, an age, distance, and reddening for such a population can be derived by isochrone fitting simultaneously in many ultraviolet (UV),

^ E-mail: george.gontcharov@tdt.edu.vn optical and infrared (IR) bands for different stages of stellar evolution, which are well recognized in the CMDs, such as the main sequence (MS), its turn-off (TO), the subgiant branch (SGB), red giant branch (RGB), horizontal branch (HB), and asymptotic giant branch (AGB). Moreover, such a fitting allows one to verify theoretical stellar evolution models underlying these isochrones.

Recently, we applied such a fitting to several CMDs of the Galactic GC NGC5904 (Gontcharov, Mosenkov \& Khovritchev 2019, hereafter Paper I). A dominant population in that cluster is evident and well separated in these CMDs. We used the accurate photometry of individual stars of NGC5904 from the Hubble Space Telescope (HST), Gaia DR2 (Gaia Collaboration et al. 2018a), Wide-field Infrared Survey Explorer (WISE, Wright et al. 2010), Sloan Digital Sky Survey (SDSS, An et al. 2008), Panoramic Survey 
Telescope and Rapid Response System (Pan-STARRS, Bernard et al. 2014), and other projects in 29 bands from the UV to mid-IR. To fit the photometric data we used the PAdova and TRieste Stellar Evolution Code (PARSEC, Bressan et al. 2012) ${ }^{1}$, the MESA Isochrones and Stellar Tracks (MIST, Paxton et al. 2011, 2013; Dotter 2016; Choi et al. 2016) ${ }^{2}$, the Dartmouth Stellar Evolution Program (DSEP, Dotter et al. 2007, 2008) ${ }^{3}$, the Bag of Stellar Tracks and Isochrones (BaSTI, Pietrinferni et al. 2004, 2006, $2013^{4}$, and the IAC-BaSTI (Hidalgo et al. 2018) ${ }^{5}$, as well as the isochrones from An et al. (2009). Adopting a spectroscopic metallicity value for NGC5904 from the literature, we derived the distance, age, and extinction law (a dependence of extinction on wavelength) for that cluster.

Paper I generally followed the approach of Hendricks et al. (2012) and Barker \& Paust (2018, hereafter BP18), but we used many more bands to draw the extinction law and more models to verify the robustness of our results. Paper I revealed that all the data and models for NGC 5904, except for some UV and SDSS data, agree with the extinction law of Cardelli, Clayton \& Mathis (1989, hereafter CCM89) with the extinction-to-reddening ratio $A_{\mathrm{V}} / E(B-V) \equiv R_{\mathrm{V}}=3.60 \pm 0.05$ and $A_{\mathrm{V}}=0.20 \pm 0.02$ mag. This extinction is twice as high as generally accepted for that GC due to a rather high extinction between 625 and $2000 \mathrm{~nm}$, i.e. between the optical and IR bands. Such a high $R_{\mathrm{V}}$ had not been supposed for the Galactic GCs before Hendricks et al. (2012) who first used IR data in their isochrone-to-data fit for the GC M4 and found a similar, rather high $R_{\mathrm{V}}=3.62 \pm 0.07$ for that cluster.

However, the large deviations of the isochrones from the data, found in Paper I for NGC 5904, can be explained not only by the high extinction and $R_{\mathrm{V}}$, but also by an intrinsic offset of the model colours due to an imperfection of the models, colour $-T_{\text {eff }}$ relations and bolometric corrections used. Hence, the approach and conclusions of Paper I should be verified for other GCs.

The aim of this study is to fit a multiband photometry and different isochrones in the CMDs for the GC NGC 6205 (M13), despite the presence of multiple populations in this cluster (Monelli et al. 2013; Savino et al. 2018). Since BP18 have investigated NGC 6205 and used partially the same photometric data (HST ACS/WFC3), we should use some additional data, especially in the IR, and pay more attention to agreement of the photometry from different sources. Following Paper I, we adopt the spectroscopic metallicity and He- $\alpha$-enhancement from the literature in order to derive the most probable age, distance and empirical extinction law of NGC 6205 by use of the photometry of its dominant population. Also, we estimate the accuracy of the isochrones under consideration.

This paper is organized as follows. We describe some key properties of NGC 6205, including metallicity, in Sect. 2. In Sect. 3 we describe the photometry used, cleaning of the datasets and creation of the fiducial sequences in the CMDs.

\footnotetext{
1 http://stev.oapd.inaf.it/cgi-bin/cmd

2 http://waps.cfa.harvard.edu/MIST/

3 http://stellar.dartmouth.edu/models/

4 http://albione.oa-teramo.inaf.it

5 http://basti-iac.oa-abruzzo.inaf.it/index.html
}

In Sect. 4 we describe the theoretical models and consequent isochrones used. Some results of our isochrone fitting are presented in Sect. 5 with an adjustment of the colours in Sect. 6. We summarize our main findings and conclusions in Sect. 7. We present an analysis of the uncertainties in Appendix A and some additional CMDs in Appendix B.

\section{SOME PROPERTIES OF NGC 6205}

NGC 6205 (M13) is one of the brightest and most wellstudied GCs of our Galaxy, located in the constellation of Hercules, at $\operatorname{RA}(2000)=16^{h} 41^{m} 41^{s}$ and $\operatorname{DEC}(2000)=$ $+36^{\circ} 27^{\prime} 36^{\prime \prime}$ (Goldsbury et al. 2010; Miocchi et al. 2013) or $l=59.0073^{\circ}$ and $b=+40.9131^{\circ}$.

We select NGC 6205 for this study due to its rich multiband photometry, low foreground and differential reddening, accurate spectroscopic estimates of its metallicity, and previous successful isochrone fitting of its dominant population, e.g. by BP18.

Bonatto, Campos \& Kepler (2013) obtained the differential reddening for this GC $\delta E(B-V)=0.033 \pm 0.09$ and $\max \delta E(B-V)=0.068$. Their note that values lower than $\delta E(B-V)<0.04$ in their analysis of many GCs may be due to photometric zero-point variations is apparently related to NGC 6205. However, Bonatto, Campos \& Kepler (2013) studied only part of the cluster's field within about 3.3 arcmin from its centre, which is covered by the HST photometry used. Recently, Bonatto (private communication) analysed NGC 6205's photometry from Stetson et al. (2019, hereafter SPZ19) and revealed that the northern and northwestern parts of a field within 16 arcmin from the cluster's centre show few hundredths magnitude higher $E(B-V)$ than the southern and south-eastern parts. This will be discussed in Sect.3.

The commonly used database of GCs by Harris $(1996)^{6}$, 2010 revision, provides for NGC 6205 a distance of $7.64 \mathrm{kpc}$, reddening $E(B-V)=0.02 \mathrm{mag}$, and apparent visual distance modulus $(m-M)_{\mathrm{V}}=14.42$. However, both distance and age estimates from the literature show a considerable diversity (BP18, and references therein).

The compiled estimate $E(B-V)=0.02$ mag by Harris (1996) is based on some original estimates, such as $E(b-$ $y)=0.015 \pm 0.010$, i.e. $E(B-V)=0.021 \pm 0.014 \mathrm{mag}$ by Grundahl, VandenBerg \& Andersen (1998), which is compared with our results in Sect. 5.1.

A recent Bayesian single-population analysis of the $H S T$ photometry in the $F 606 W$ and $F 814 W$ bands by Wagner-Kaiser et al. (2017) gives $[\mathrm{Fe} / \mathrm{H}]=-1.53,(m-M)_{\mathrm{V}}=$ $14.442_{-0.005}^{+0.006}$, an age of $13.094_{-0.082}^{+0.084}, A_{\mathrm{V}}=0.12_{-0.006}^{+0.005}$, and, consequently, $E(B-V)=0.039 \pm 0.002 \mathrm{mag}$ by use of the extinction law of CCM89 with $R_{\mathrm{V}}=3.1$. Moreover, analysing 69 GCs, Wagner-Kaiser et al. (2017) note, 'In many cases, we estimate moderately larger absorption than the values from Harris (2010)'.

A recent isochrone fitting of $V I$ photometry by Deras et al. (2019) gives $E(B-V)$, age and distance of $0.02 \mathrm{mag}, 12.6 \mathrm{Gyr}$ and $7.1 \mathrm{kpc}$, respectively. However,

\footnotetext{
6 https://www.physics.mcmaster.ca/ ${ }^{\sim}$ harris/mwgc. dat
} 
Deras et al. (2019) note that the HB needs $E(B-V)=0.04$ mag for a better fit.

We adopt the spectroscopic estimate of metallicity $[\mathrm{Fe} / \mathrm{H}]=-1.58 \pm 0.04$ (equal to $Z=0.0004$ with the solar metallicity $Z=0.0152$ ) derived for NGC 6205 by Carretta et al. (2009) within their abundance scale, which is well defined from a robust dataset of $[\mathrm{Fe} / \mathrm{H}]$ abundances of GCs. This estimate agrees with recent estimates from a Fourier decomposition of the light curves of RRab and RRc stars by Deras et al. (2019), $[\mathrm{Fe} / \mathrm{H}]=-1.58 \pm 0.09$, and from a review of chemical composition of Galactic GCs by Marsakov, Koval' \& Gozha (2019), $[\mathrm{Fe} / \mathrm{H}]=-1.54 \pm 0.06$.

The estimate of metallicity from spectroscopy is much more accurate than that from photometry. Hence, we do not determine metallicity from our isochrone fitting in the CMDs. However, we check a range of metallicity, outside of which the derived cluster's characteristics and the isochrone fitting, in particular, the tilt of the RGB, become completely unreliable; this range is $-1.38<[\mathrm{Fe} / \mathrm{H}]<-1.78$. Thus, the results of this study do not contradict the adopted metallicity.

Marino et al. (2019); Marsakov, Koval' \& Gozha (2019) find an enhanced average $\alpha$ abundance of $[\alpha / \mathrm{Fe}] \approx 0.2$ for NGC6205, with noticeable variations from star to star and from element to element. Denissenkov et al. (2015); Cohen et al. (2017); Denissenkov (2017); Wagner-Kaiser et al. (2017) show a significant $(0.30<Y<0.38)$ helium enhancement in NGC 6205 . Denissenkov et al. (2015) note three populations of its stars: with normal $(Y=0.25)$, intermediate $(Y=0.33)$, and extreme $(Y=0.38)$ compositions, with a dominance of the intermediate one with $>60$ per cent of the stars. Based on Strömgren photometry and its comparison with several spectroscopic studies, Savino et al. (2018, hereafter SMB18) conclude that about 80 per cent of giant stars of NGC 6205 belong to the He- $\alpha$-enriched population. Such a clear domination of the population makes the photometry of this cluster quite convenient for an isochrone fitting.

Since $\alpha$ and helium enhancements make isochrones with the same $[\mathrm{Fe} / \mathrm{H}]$ redder and bluer, respectively (see Paper I for details), a cumulative effect of both the enhancements may keep the isochrones close to the solar-scaled ones. In Sect. 5 we will test the difference between the solar-scaled and $\mathrm{He}-\alpha$-enhanced isochrones and, consequently, the importance of taking into account the $\mathrm{He}-\alpha$-enhancement. The contribution of the uncertainty due to the adopted $\mathrm{He}-\alpha-$ enhancement to the total uncertainty is discussed in Appendix A.

\section{PHOTOMETRY}

We use the following datasets of photometry in 34 filters in order to create CMDs for NGC 6205:

- 54814 stars $^{7}$ with photometry in the $F 275 W, F 336 W$, F438W filters from the HST Wide Field Camera 3 (WFC3) UV Legacy Survey of Galactic Globular Clusters and the F606W , F814W filters from its Advanced Camera for Surveys

\footnotetext{
7 The original datasets typically contain many more stars. Here we provide the number of stars after rejecting the poor photometry, as described in Sect. 3.1.
}

(ACS) survey of Galactic globular clusters (Nardiello et al. $2018)^{8}$,

- 25032 stars with the SDSS photometry in the ugriz filters and related fiducial sequences from An et al. (2008, 2009) ${ }^{9}$,

- 23023 stars with $U B V I$ photometry (SPZ19) ${ }^{10}$,

- 18685 stars with Strömgren $v y$ photometry from the Isaac Newton Telescope - Wide Field Camera (INT/WFC) (SMB18) ${ }^{11}$,

- 12868 stars with Strömgren uvby photometry from the Nordic Optical Telescope (NOT) on La Palma, Canary Islands (Grundahl, VandenBerg \& Andersen 1998, hereafter GVA98),

- 6747 stars with photometry from Piotto et al. (2002) in the $F 439 W$ and $F 555 W$ filters from the HST Wide Field and Planetary Camera 2 (WFPC2),

- 4530 stars with Gaia DR2 photometry in the $G, G_{\mathrm{BP}}$ and $G_{\mathrm{RP}}$ filters from Bustos Fierro \& Calderòn (2019) ${ }^{12}$,

- 2152 stars and fiducial sequences derived by Rey et al. (2001) in the $B V$ bands, based on the Michigan-DartmouthMIT (MDM) Observatory $2.4 \mathrm{~m}$ telescope ${ }^{13}$,

- 1699 stars with Wide-field Infrared Survey Explorer (WISE) photometry in the $W 1$ filter from unWISE catalogue (Schlafly et al. 2019), which are cross-identified by us with the other lists under consideration,

- the fiducial sequences derived by Clem, VandenBerg \& Stetson (2008) in the $u^{\prime} g^{\prime} r^{\prime} i^{\prime} z^{\prime}$ bands with the MegaCam wide-field imager on the Canada-France-Hawaii Telescope (CFHT/MegaCam),

- the fiducial sequence derived by Paltrinieri et al. (1998, hereafter PFF98) for the $B-V$ colour based on observations with the $1.23-\mathrm{m}$ telescope at the German-Spanish Astronomical Center, Calar Alto, Spain (hereafter GSAC 1.23-m),

- the fiducial sequences derived by Brasseur et al. (2010, hereafter BSV10) for the $V-J$ and $V-K$ colours based on IR observations with the WIRCam imager on the Canada-France-Hawaii Telescope (CFHT/WIRCam), calibrated by use of the Two Micron All-Sky Survey (2MASS, Skrutskie et al. 2006) stars, and combined with an early version of the $V$-band data from SPZ19,

- the fiducial sequences derived by Bernard et al. (2014) in the $g_{\mathrm{P} 1}, r_{\mathrm{P} 1}, i_{\mathrm{P} 1}, z_{\mathrm{P} 1}$, and $y_{\mathrm{P} 1}$ bands of Pan-STARRS DR1.

Table 1 presents the effective wavelength $\lambda_{\text {eff }}$ in $\mathrm{nm}$ and the median precision of the photometry for each filter.

Each star has a photometry in some but not all filters.

We cross-identify some of the datasets with each other.

\footnotetext{
8 http://groups.dfa.unipd.it/ESPG/treasury.php

9 http://classic.sdss.org/dr6/products/value_added/anjohnson08_cluste Their fiducial sequences do not expand on the HB. Therefore, we calculate our own fiducial sequences for the HB by use of these data, however, for the RGB, SGB, TO and MS our fiducial sequences appear to coincide with those of An et al. (2008, 2009) within \pm 0.01 mag.

10 http://cdsarc.u-strasbg.fr/viz-bin/cat/J/MNRAS/485/3042 We do not use the photometry in the $R$ filter due to its very low precision.

11 We do not use the photometry from SMB18 in the Strömgren $b$ band due to a problem with the data, which is noted by the authors.

12 http://cdsarc.u-strasbg.fr/viz-bin/cat/J/MNRAS/488/3024

13 http://cdsarc.u-strasbg.fr/viz-bin/cat/J/AJ/122/3219
} 
Table 1. The effective wavelength $(\mathrm{nm})$ and median precision of the photometry (mag) for the filters under consideration.

\begin{tabular}{|c|c|c|c|}
\hline Telescope & Filter & $\lambda_{\mathrm{eff}}$ & Median precisior \\
\hline$H S T / \mathrm{WFC} 3$ & $F 275 W$ & 274 & 0.02 \\
\hline$H S T / \mathrm{WFC} 3$ & $F 336 W$ & 329 & 0.01 \\
\hline INT/WFC (SMB18) & Strömgren $u$ & 347 & 0.02 \\
\hline NOT (GVA98) & Strömgren $u$ & 347 & 0.02 \\
\hline Various (SPZ19) & $U$ & 354 & 0.01 \\
\hline SDSS & $u$ & 360 & 0.06 \\
\hline INT/WFC (SMB18) & Strömgren $v$ & 412 & 0.01 \\
\hline NOT (GVA98) & Strömgren $v$ & 412 & 0.01 \\
\hline$H S T / W F P C 2$ & $F 439 W$ & 435 & 0.03 \\
\hline Various (SPZ19) & $B$ & 437 & 0.01 \\
\hline MDM (Rey et al. 2001) & $B$ & 437 & 0.01 \\
\hline GSAC $1.23-\mathrm{m}$ (PFF98) & $B$ & 437 & 0.05 \\
\hline$H S T / \mathrm{WFC} 3$ & $F 438 W$ & 437 & 0.01 \\
\hline NOT (GVA98) & Strömgren $b$ & 467 & 0.01 \\
\hline SDSS & $g$ & 471 & 0.02 \\
\hline Pan-STARRS1 & $g_{\mathrm{P} 1}$ & 480 & 0.02 \\
\hline CFHT/MegaCam & $g^{\prime}$ & 491 & 0.02 \\
\hline Gaia DR2 & $G_{\mathrm{BP}}$ & 539 & 0.03 \\
\hline Various (SPZ19) & $V$ & 547 & 0.01 \\
\hline MDM (Rey et al. 2001) & $V$ & 547 & 0.01 \\
\hline GSAC 1.23-m (PFF98) & $V$ & 547 & 0.05 \\
\hline INT/WFC (SMB18) & Strömgren $y$ & 548 & 0.02 \\
\hline NOT (GVA98) & Strömgren $y$ & 548 & 0.02 \\
\hline$H S T / \mathrm{WFPC} 2$ & $F 555 W$ & 549 & 0.08 \\
\hline$H S T / \mathrm{ACS}$ & $F 606 W$ & 588 & 0.01 \\
\hline Pan-STARRS1 & $r_{\mathrm{P} 1}$ & 620 & 0.02 \\
\hline SDSS & $r$ & 621 & 0.03 \\
\hline CFHT/MegaCam & $r^{\prime}$ & 625 & 0.02 \\
\hline Gaia DR2 & $G$ & 642 & 0.02 \\
\hline SDSS & $i$ & 743 & 0.04 \\
\hline Pan-STARRS1 & $i_{\mathrm{P} 1}$ & 746 & 0.02 \\
\hline Gaia DR2 & $G_{\mathrm{RP}}$ & 767 & 0.03 \\
\hline CFHT/MegaCam & $i^{\prime}$ & 767 & 0.02 \\
\hline$H S T / \mathrm{ACS}$ & $F 814 W$ & 794 & 0.01 \\
\hline Various (SPZ19) & I & 812 & 0.01 \\
\hline Pan-STARRS1 & $z_{\mathrm{P} 1}$ & 860 & 0.02 \\
\hline CFHT/MegaCam & $z^{\prime}$ & 885 & 0.02 \\
\hline SDSS & $z$ & 885 & 0.06 \\
\hline CFHT/WIRCam, 2MASS & $J$ & 1229 & 0.02 \\
\hline CFHT/WIRCam, 2MASS & $K$ & 2147 & 0.02 \\
\hline WISE & $W 1$ & 3316 & 0.01 \\
\hline
\end{tabular}

The cross-identification in the UV and optical ranges is done to estimate possible offsets and systematic errors of the photometry of the different datasets, as discussed in Sect. 6 (as a result, we decide to use the Strömgren datasets by SMB18 and GVA98 together due to their negligible photometric differences for common stars). The cross-identification with unWISE is done to determine some IR extinction zero-points in Sect. 5.1 by use of the optical-IR colours and reddenings, following the approach of Paper I.

The following datasets have an IR extinction zero-point: $U B V I$ by SPZ19 based on the derived reddening $E(I-W 1)$ or $E(V-W 1), B V$ by Rey et al. (2001) based on the derived $E(V-W 1)$, Gaia based on the derived $E(G-W 1)$ or $E\left(G_{\mathrm{RP}}-W 1\right)$, SDSS based on the derived $E(z-W 1)$, Strömgren by SMB18 and GVA98 based on the derived $E(y-W 1)$, and $V J K$ by BSV10 based on the derived $E(V-$

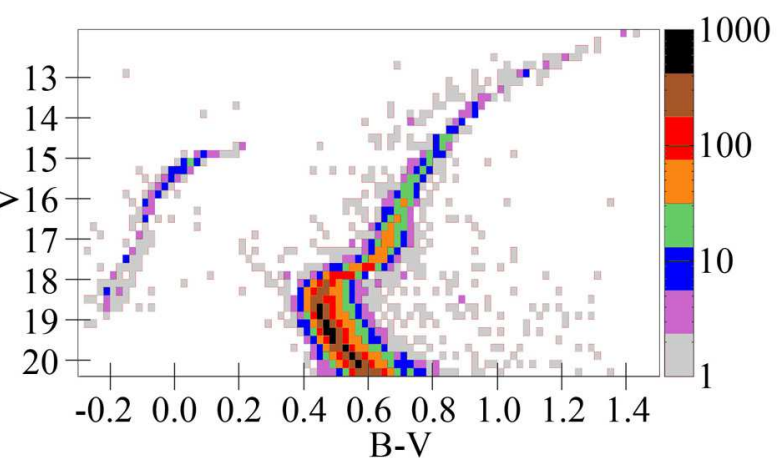

Figure 1. The distribution of 23023 stars from SPZ19 in the colour-magnitude bins of $0.02 \times 0.2 \mathrm{mag}$ on the $B-V$ versus $V$ CMD of NGC 6205. The number of the stars in each bin is shown by the colour scale on the right.

$K)$. The remaining datasets, including all the cases when we have fiducial sequences only, without data for individual stars, have not been tied to an IR photometric catalogue: HST ACS/WFC3, HST WFPC2, BV by PFF98, PanSTARRS by Bernard et al. (2014), and CFHT/MegaCam by Clem, VandenBerg \& Stetson (2008). Their extinction zeropoints are defined by the CCM89 extinction law with $R_{\mathrm{V}}$ determined from the datasets with an IR extinction zeropoint, as described in Sect. 5.1.

These vast amount of photometric data make it possible to consider and fit the isochrones to dozens of CMDs with different colours. They cover a wide wavelength range between the UV and middle IR. Each CMD provides us with independent estimates of age and distance, while each dataset gives us an irrespective set of the derived reddenings. Such a set draws an empirical extinction law for a dataset with an IR extinction zero-point, while each dataset without such a zero-point can be used as a set of reddenings to confirm or reject this law.

To fit by isochrones, a fiducial sequence must be determined in each CMD as a colour-magnitude relation for single stars of a dominant population in a cluster. We calculate the fiducial sequence for each CMD as a locus of the number density maxima in some colour-magnitude bins.

An example of the distribution of the stars in a CMD, after the cleaning for poor photometry described in Sect. 3.1, is shown in Fig. 1: $B-V$ versus $V$ CMD with the colourmagnitude bins of $0.02 \times 0.2 \mathrm{mag}$. This is an optimal bin size for a rather dense distribution. It is evident from Fig. 1 that in such a case a fiducial sequence can be derived, at least, with a precision of half of one bin. For a less dense distribution (for example, in the CMDs with the $W 1$ band) the bin size increases to $0.04 \times 0.4 \mathrm{mag}$. Consequently, it is evident that an average fiducial sequence colour (averaged over > 15 CMD magnitude bins and finally transformed into the reddening in a comparison with an isochrone) can be determined with a precision better than $0.04 / 2 / 15^{0.5} \approx 0.005$ mag. An example fiducial sequence for $V$ versus $B-V$ is presented in Table 2. All other fiducial sequences can be provided on request.

Since we use a similar number of stars for all the filters of a dataset, and since we need a similar and rather large number of stars in the bins of number density maxima to obtain a precise fiducial sequence position, the optimal bin 


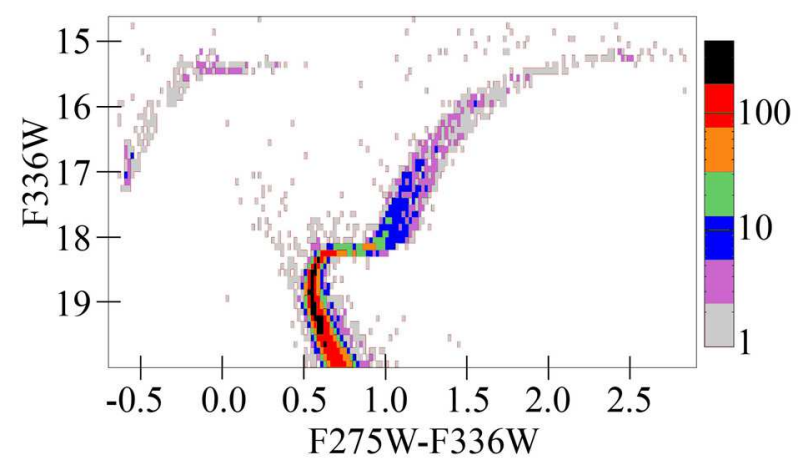

Figure 2. The distribution of 24303 stars with the $F 275 W$ and F336W HST WFC3 photometry in the colour-magnitude bins of $0.02 \times 0.1 \mathrm{mag}$ on the $F 275 W-F 336 W$ versus $F 336 W$ CMD of NGC 6205. The number of stars in each bin is shown by the colour scale on the right.

Table 2. The fiducial sequence for NGC $6205 V$ versus $B-V$ based on the data of SPZ19. The complete table is available online.

\begin{tabular}{rr}
\hline$V$ & $B-V$ \\
\hline 18.6 & -0.210 \\
18.4 & -0.190 \\
18.2 & -0.170 \\
18.0 & -0.150 \\
17.7 & -0.130 \\
$\cdots$ & $\cdots$ \\
\hline
\end{tabular}

size depends on the wavelength range used. For example, if we consider a bin of $0.02 \mathrm{mag}$ for both the $B-V$ and $V-I$ colours, we should use a bin size of $0.04 \mathrm{mag}$ for the $B-I$ colour.

Similar to NGC 5904 in Paper I, we can easily define the fiducial sequences for NGC 6205 due to (i) the high degree of completeness of the stellar samples under consideration, at least between the HB and TO, (ii) low differential reddening, (iii) low contamination from foreground/background stars at such a high latitude, and (iv) the high percentage of the dominant population stars.

UV CMDs are difficult to use for isochrone fitting, particularly due to an emphasizing of the colour differences between the stellar populations by some combinations of UV filters (Monelli et al. 2013; Barker \& Paust 2018; Savino et al. 2018). However, it is interesting to explore with recent data and isochrones - which combination of the UV filters makes the difference between the populations being negligible or allows us to derive robust properties of a dominant population. Therefore, we use UV filters in our study as well.

A UV CMD, after cleaning for poor photometry in Sect. 3.1, is shown in Fig. 2. It illustrates a separation of the multiple populations only for the RGB, making it wider. This makes a different contribution to the uncertainties of the derived reddening, distance and age.

A dominant population with about 80 per cent of the stars, as predicted by SMB18, formes a peak of the stel- lar distribution along the bluer side of the RGB. This peak has a width up to four colour bins (i.e. $0.08 \mathrm{mag}$ ) in the cross-sections of the RGB along the colour. Hence, the fiducial sequence colour in such a cross-section (i.e. within a magnitude bin) can be determined with a precision of half of the peak width, i.e., at least, \pm 0.04 mag. To derive the reddening, a fiducial sequence colour, averaged over many magnitude bins, is used. For example, for the CMD in Fig. 2 we use 40 magnitude bins within $15.5<F 336 W<19.5$ mag. In each magnitude bin we have an independent estimate of the fiducial colour. Hence, a typical mean fiducial sequence colour uncertainty is $0.04 / 40^{0.5}=0.006$ mag.

To derive the distance, the magnitudes of the $\mathrm{HB}$ and $\mathrm{SGB}$ are used. The CMD in Fig. 2 is typical showing no influence of the multiple populations on the HB or SGB magnitude and, consequently, on the distance. The same is true for the age, when it is obtained from the HB-SGB magnitude difference. However, when it is obtained from the SGB length, it can be affected by a separation of the multiple populations at the base of the RGB. The CMD in Fig. 2 shows an uncertainty of the SGB length about half of the colour bin, i.e. $\pm 0.01 \mathrm{mag}$. This uncertainty is typical in the UV, decreasing in the optical and IR range. This corresponds to an age uncertainty of \pm 0.5 Gyr. It gives a minor contribution w.r.t. the other uncertainties.

Finally, we find a rather precise positioning of the dominant population in all the CMDs. The largest uncertainty 0.01 mag for the fiducial sequence colour, due to the multiple populations, is found for the $u-v$ versus $v$ CMD with the dataset of GVA98.

These uncertainties due to the existence of multiple populations in NGC 6205 are taken into account in the balance of uncertainties, presented in Appendix A.

\subsection{Cleaning the datasets}

In this section we describe how we clean the original datasets from poor photometry.

We analyse the distribution of stars on the precision of their photometry and only select stars with errors of less than $0.07 \mathrm{mag}$ in the HST ACS and WFC3 colours, $0.07 \mathrm{mag}$ in Rey et al. (2001)'s colour, $0.08 \mathrm{mag}$ in the Gaia colour, $0.10 \mathrm{mag}$ in the $U B V I$ colours, and $0.15 \mathrm{mag}$ in the remaining colours. However, the median precision of the colours is much higher, as is evident from Table 1. Also, for each colour we only select stars brighter than a magnitude limit, in which 97 per cent of stars have a photometric error less than the above-mentioned limits. Typically, this means a magnitude limit of about $<20.5$ mag for the optical filters. These magnitude cuts allow us to consider only the magnitude ranges where there is no bias of the fiducial sequence due to the removal of stars with poor photometry.

In addition, we use some photometric quality parameters to clean the datasets from poor photometry. We present here only some interesting cases of such cleaning.

Initially we select 43714 Gaia DR2 stars in the field of NGC6205 by use of the catalogue of Bustos Fierro \& Calderòn (2019). However, only 26649 stars among these have photometry in all three Gaia bands, which is needed to estimate the photometric quality. Moreover, only 4844 stars among them follow the photometric 


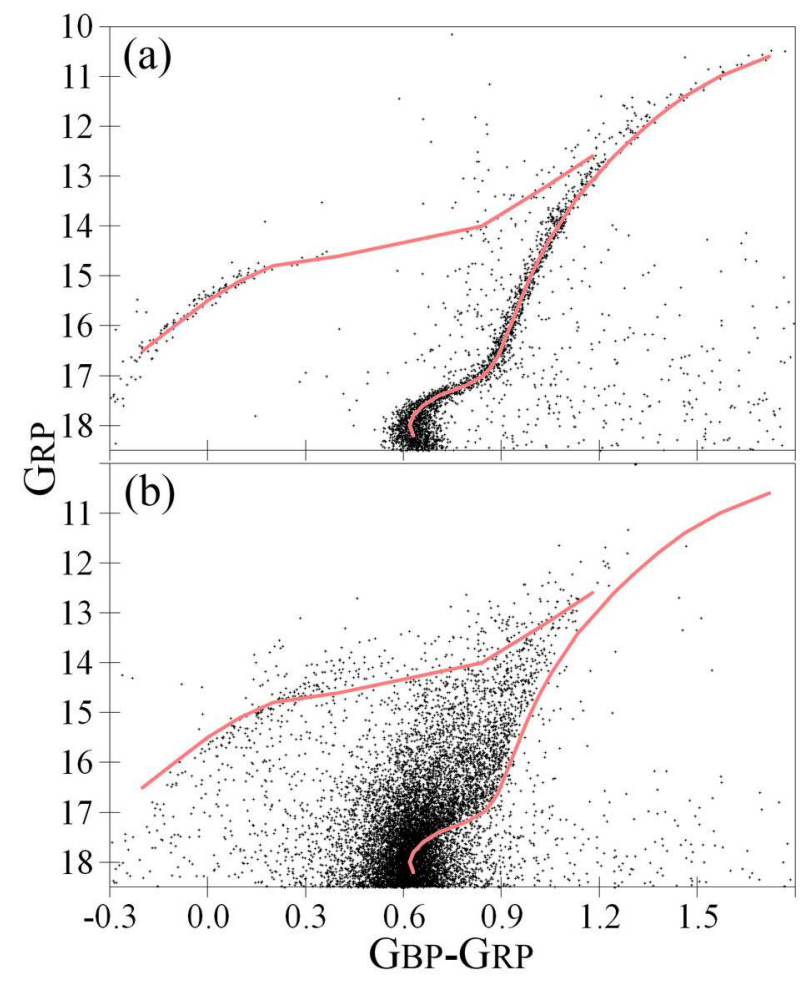

Figure 3. $G_{\mathrm{BP}}-G_{\mathrm{RP}}$ versus $G_{\mathrm{RP}} \mathrm{CMD}$ of NGC 6205 for (a) 4844 and (b) 21805 stars, which follow the limit (1) or do not, respectively. The fiducial sequence is shown by the curve as a reference.

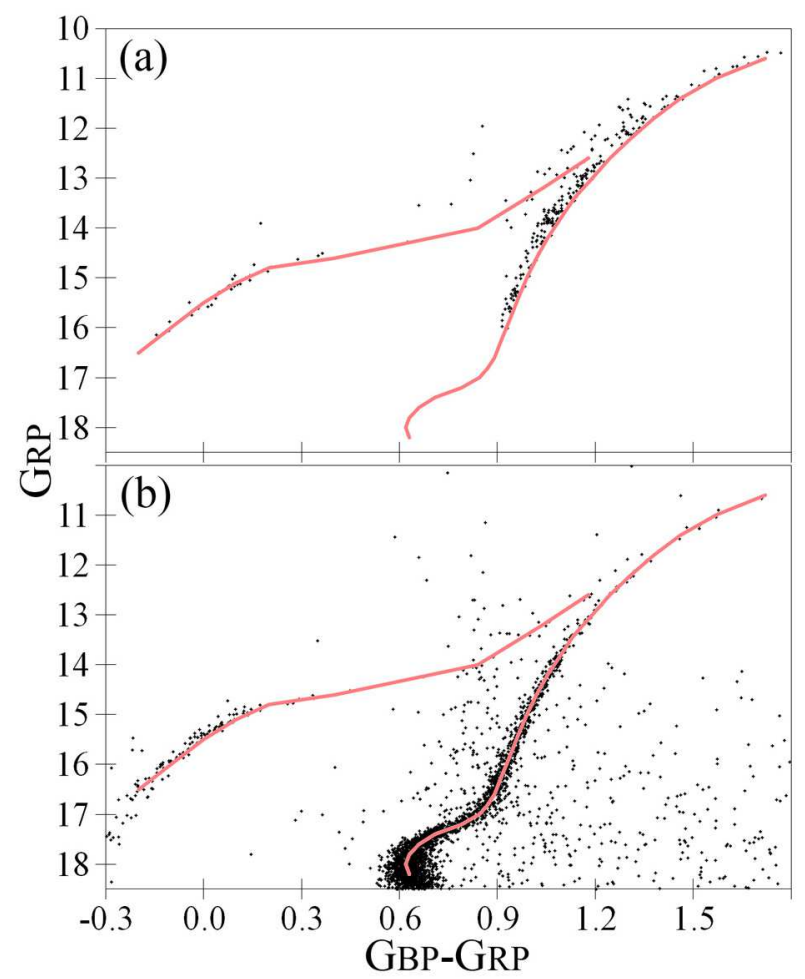

Figure 4. The same as Fig. 3 but for (a) 314 stars within and (b) 4530 stars outside the 2.7 arcmin radius from the cluster's centre. The fiducial sequence is shown by the curve as a reference. quality criterion, suggesting that they are 'well-behaved single sources' (Gaia Collaboration et al. 2018b):

$$
\begin{gathered}
\text { phot_bp_rp_excess_factor } \equiv \\
\equiv\left(F_{\mathrm{BP}}-F_{\mathrm{RP}}\right) / F_{\mathrm{G}}<1.3+0.06\left(G_{\mathrm{BP}}-G_{\mathrm{RP}}\right)^{2},
\end{gathered}
$$

where $F_{\mathrm{BP}}, F_{\mathrm{RP}}$, and $F_{\mathrm{G}}$ are the fluxes within the Gaia filters.

To justify the removal of such a large amount of stars, we compare the CMDs with the subsamples separated by the limit (1) in Fig. 3. It is seen that any relaxing of the limit (1) would lead to a systematic blueward colour offset of the derived fiducial. Thus, phot_bp_rp_excess_factor appears to be a useful photometric quality parameter in order to separate stars with good or bad photometry in such studies of globular clusters.

However, the resulting dataset of the Gaia DR2 photometry still contains a number of stars with erroneous photometry in the most crowded centre of the cluster. This is seen in Fig. 4, where 314 stars within 2.7 arcmin from the centre and the remaining 4530 stars within 16 arcmin from the centre are shown in plots (a) and (b), respectively: a systematic colour offset of the observed RGB by about 0.02 mag is evident in plot (a) w.r.t. (b). These 314 stars dominate at brighter parts of the RGB and AGB, higher than a point where they meet each other, i.e. at $G_{\mathrm{RP}}<14$. Therefore, we do not use these stars and these brighter parts of the RGB and AGB in calculating the fiducial sequence and in the fiducial-isochrone fitting. This case indicates that the Gaia DR2 photometry may be unacceptable in central regions of GCs.

Note that such poor photometry is typical for brighter parts of the RGB and AGB of all the datasets under consideration. Therefore, we do not use these RGB and AGB parts in our isochrone fitting for all the datasets.

For the dataset of SPZ19 there are several quality parameters to be taken into account to separate stars with accurate photometry. In the field of NGC 6205 we remove more than 40000 stars with DAOPHOT sharp parameter $\mid$ sharp $\mid>0.3$. In addition, we remove 4767 stars with a variability evidence $>$ 9, which is defined by SPZ19 as the logarithm of the Welch-Stetson variability index (Welch \& Stetson 1993) times its weight. These removed stars are shown in Fig. 5 (a), while the remaining 23023 stars are in plot (b), with their precise distribution shown in Fig. 1. It is seen that the removed stars would provide a noticeable redward bias of the fiducial.

The resulting CMDs for the stars within and outside the 2.4 arcmin radius from the cluster's centre are shown in Fig. 6. Although a lot of faint stars are lost in the cluster's centre, the remaining stars demonstrate only a larger scatter around the fiducial sequence, while the systematic blueward colour offset of the remaining RGB stars in the centre w.r.t. the periphery is less than $0.01 \mathrm{mag}$. Such a colour offset may be due to a lower reddening in the centre, in agreement with the results of Bonatto (private communication). This shows that any centre-periphery distinction cannot be separated from the differential reddening.

Fig. 7 shows the differential reddening for the SPZ19 dataset within 25 arcmin from the cluster's centre. It is seen that both the RGB and MS are about 0.02 mag redder in the north-western than in the south-eastern corner of the NGC 6205's field. This agrees with the differential reddening found by Bonatto (private communication) and mentioned 


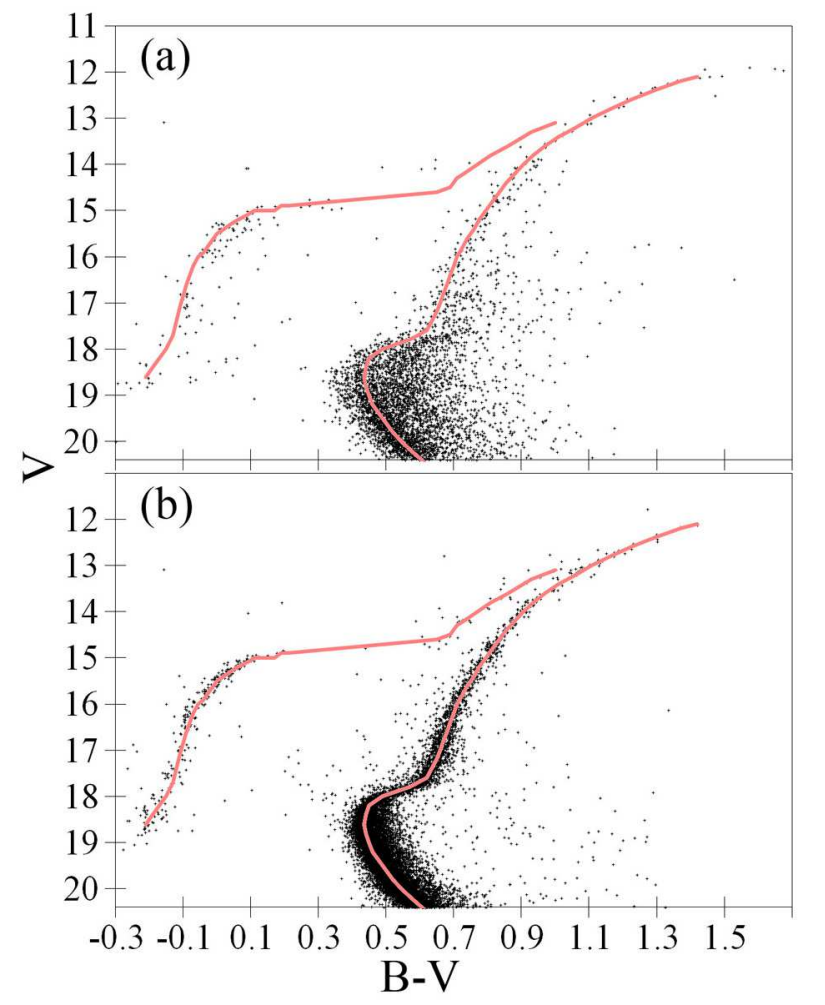

Figure 5. $B-V$ versus $V$ CMD of NGC 6205 based on the data from SPZ19 for (a) 4767 stars with variability evidence $>9$ and (b) 23023 remaining stars. The fiducial sequence is shown by the curve as a reference.

in Sect. 1. Therefore, it seems that the differential reddening dominates among the field effects after the cleaning of the SPZ19 dataset. However, for other datasets and CMDs, such as the HST ACS/WFC3 dataset, a combination of the field effects may be more complex.

From the HST ACS/WFC3 dataset, we only use stars with $\mid$ sharp $\mid<0.15$, following Nardiello et al. (2018). This removes the majority of stars from the original dataset. Also, we remove stars with a membership probability $<90$ per cent. However, we use stars with the undefined probability, since they make up a significant portion of the sample and do not show a systematic offset in any CMD. Finally, we only use stars with a quality-fitting parameter $>0.9$.

However, the resulting sample of 54814 stars still demonstrates some systematic effects, even in the small HST field of $3.5 \times 3.5$ arcmin. Fig. 8 shows the $F 606 W-F 814 W$ versus $F 814 W$ CMD for the stars within and outside the 1.14 arcmin radius from the cluster's centre in plots (a) and (b), respectively. We show only a central part of the CMD, where the effects are noticeable (they are negligible at the $\mathrm{HB}$ and SGB). The best-fitting IAC-BaSTI isochrone is shown as a reference. It is seen that in the centre of the cluster's field the RGB is bluer, while the MS is redder than in the periphery of the field. This may be explained only by a combination of effects, such as a crowding effect, differential reddening, different representation of the multiple populations in different parts of the cluster, a systematic error of photometry over the field, a variable contamination by field stars, and/or an incompleteness of the sample. The latter is especially noticeable for the faintest stars $(F 814 W>20 \mathrm{mag})$ in Fig. 8 (a)
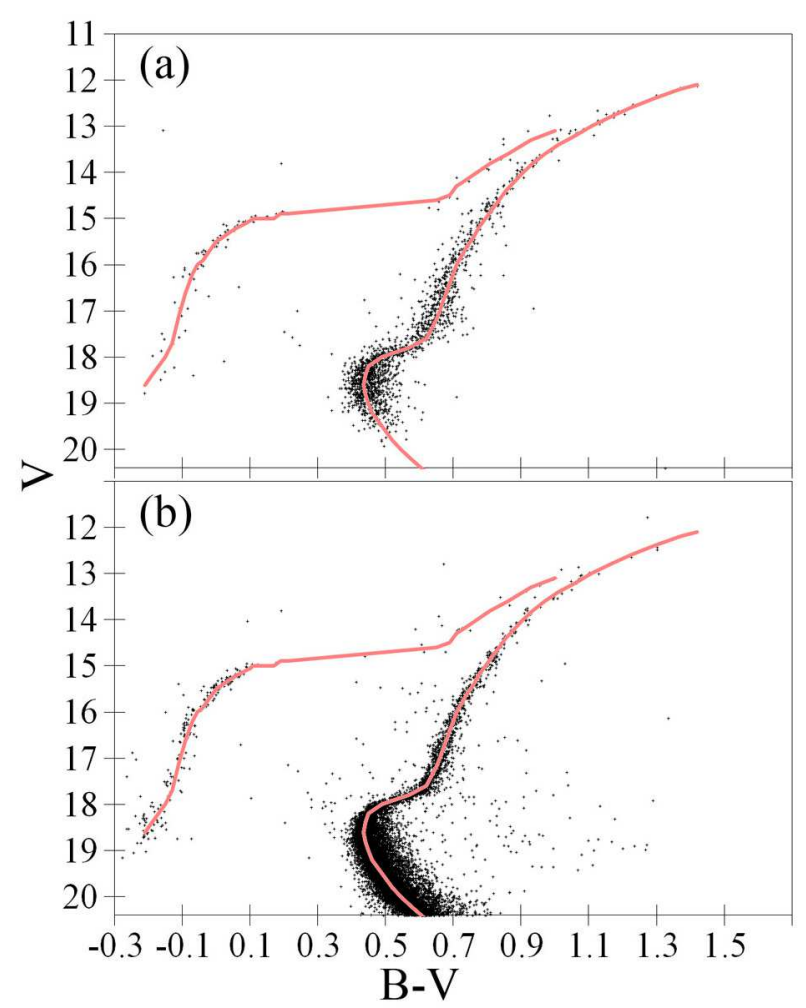

Figure 6. The same as Fig. 5 but for (a) 1852 stars within and (b) 21171 stars outside the 2.4 arcmin radius from the cluster's centre. The fiducial sequence is shown by the curve as a reference.

w.r.t. (b), i.e. in the centre w.r.t. the periphery, apparently, due to a crowding effect. Thus, these effects are difficult to separate and have to be considered mutually.

We briefly analyse these field effects for all the CMDs, which are based on the largest datasets of HST ACS/WFC3, SDSS, SPZ19, GVA98, and SMB18. First, we compare partial CMDs, i.e. the ones for different parts of the field, by use of a moving window with about 5000 stars to calculate a partial fiducial sequence for each partial CMD. The number of stars in the moving window varies slightly depending on their distribution over the field. We consider the shifts of the partial fiducial sequences w.r.t each other along the reddening vector as estimates of the reddening and related field effects in different parts of the field. This approach allows us to reveal only a gradient, but not a detailed map of the field effects. It seems to be enough, since we find a rather small field effect at a level of $\delta E(B-V)<0.02$ mag for all the CMDs. This agrees with the differential reddening results of Bonatto, Campos \& Kepler (2013) and Bonatto (pivate communication). Given such a small effect for the largest datasets, we suggest a similar one for the remaining datasets. Also, we check that stars from the areas with bluer and redder partial fiducial sequences are equally represented in all the CMDs for all the datasets, or, at least, that an uneven distribution of stars does not introduce a field effect bias more than 0.005 mag into an average fiducial sequence colour. We find that, indeed, this is so for all the datasets.

We can conclude that a typical variation of the fiducial sequence colour over the field, including the differential reddening, is at a level of $\pm 0.01 \mathrm{mag}$, e.g. as between plots (a) 


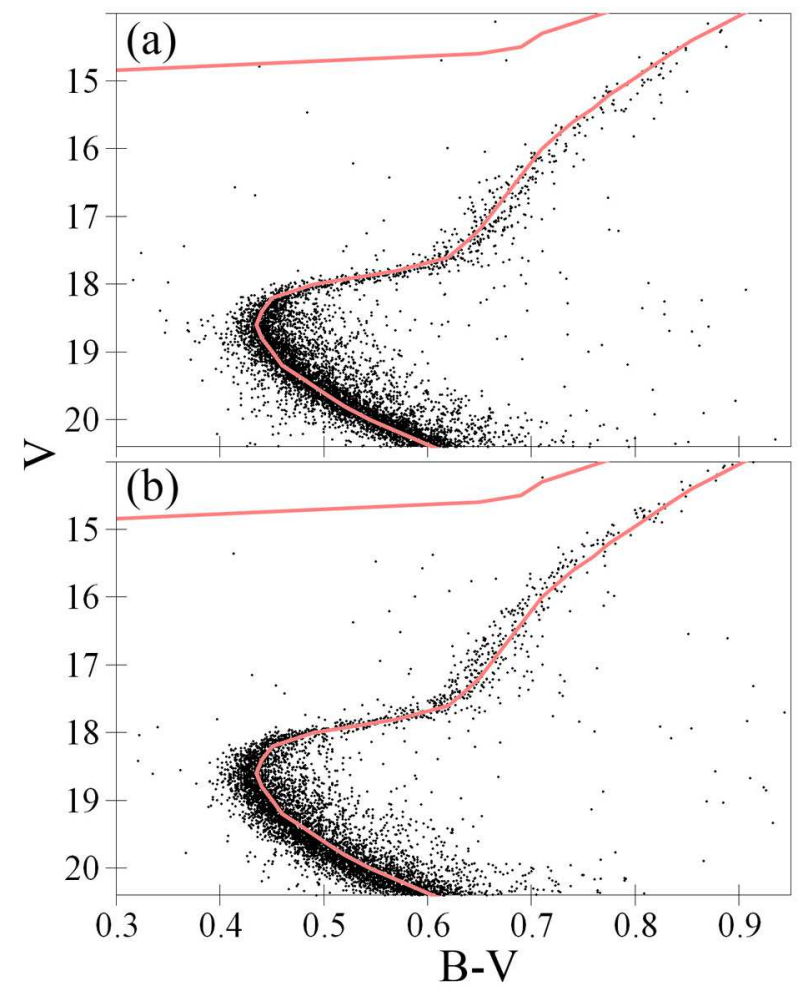

Figure 7. The same as Fig. 5 but for (a) 8130 stars in the northwest $(Y-X>164$ pixels in the SPZ19's coordinate frame) and (b) 7970 stars in the south-east $(Y-X<-167$ pixels in the SPZ19's coordinate frame) corner of NGC 6205's field. The fiducial sequence is shown by the curve as a reference.

and (b) in Fig. 8. Since we consider the whole cluster field, where this effect is averaged, it gives a fiducial sequence colour offset at a level of $\pm 0.005 \mathrm{mag}$. This offset is even lower in the cases when we use a fiducial sequence presented by the dataset's authors after an investigation of the field errors by them.

\section{THEORETICAL MODELS AND ISOCHRONES}

In order to fit the CMDs of NGC6205, we use the following theoretical models of stellar evolution and related isochrones:

- PARSEC together with the COLIBRI PR16 (Marigo et al. 2013; Rosenfield et al. 2016) assuming $[\mathrm{Fe} / \mathrm{H}]=-1.58, Z=0.0004, Y=0.2485+1.78 Z=0.2492$, $[\alpha / \mathrm{Fe}]=0$, solar $Z=0.0152$, mass loss efficiency $\eta=0.2$, where $\eta$ is the free parameter in Reimers' law (Reimers 1975);

- MIST with the average observed photospheric metallicity $[\mathrm{Fe} / \mathrm{H}]=-1.58$, which corresponds to the initial protostar birth cloud bulk metallicity $[\mathrm{Fe} / \mathrm{H}]=-1.61, Z=0.000365$, $Y=0.2495,[\alpha / \mathrm{Fe}]=0$ w.r.t. the protosolar $Z=0.0142$;

- DSEP, solar-scaled abundance (hereafter solar-scaled DSEP) with $[\mathrm{Fe} / \mathrm{H}]=-1.58, Z=0.00045, Y=0.2457$, $[\alpha / \mathrm{Fe}]=0$ and enhanced abundance (hereafter enhanced DSEP) with $[\mathrm{Fe} / \mathrm{H}]=-1.58, Z=0.00078, Y=0.33,[\alpha / \mathrm{Fe}]=$ +0.40 , both with the solar $Z=0.0189$;

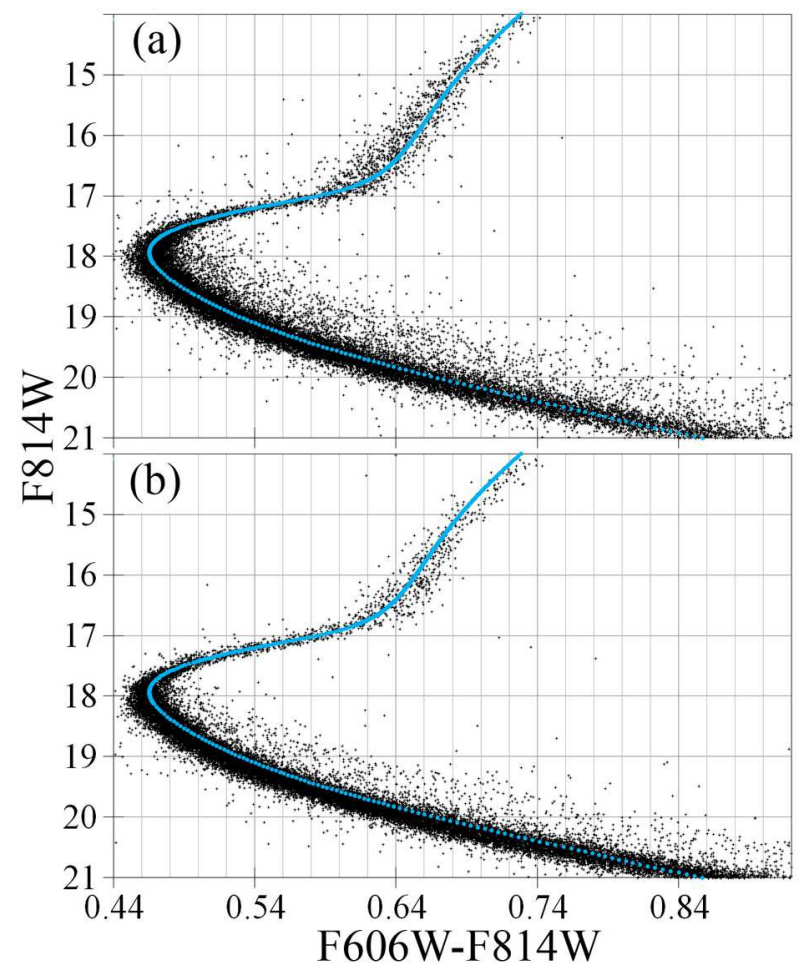

Figure 8. $F 606 W-F 814 W$ versus $F 814 W$ CMD of NGC 6205 based on the HST ACS data for (a) 25231 stars within and (b) 24958 stars outside the 1.14 arcmin radius from the cluster's centre. The best-fitting IAC-BaSTI isochrone is shown by the curve as a reference.

- BaSTI, solar-scaled abundance (hereafter solar-scaled BaSTI) with $[\mathrm{Fe} / \mathrm{H}]=-1.614, Z=0.0004, Y=0.245,[\alpha / \mathrm{Fe}]=$ 0 , mass loss efficiency $\eta=0.4$ and enhanced abundance (hereafter enhanced BaSTI) with $[\mathrm{M} / \mathrm{H}]=-1.27,[\mathrm{Fe} / \mathrm{H}]=$ $-1.62, Z=0.0009, Y=0.3,[\alpha / \mathrm{Fe}]=+0.4$, mass loss efficiency $\eta=0.2$, both with the solar $Z=0.019$;

- IAC-BaSTI with $[\mathrm{Fe} / \mathrm{H}]=-1.58, Z=0.0004, Y=0.2476$, $[\alpha / \mathrm{Fe}]=0$, overshooting, diffusion, mass loss efficiency $\eta=$ $0.3^{14}$ and the initial solar $Z=0.0172$ and $Y=0.2695$.

The current releases of PARSEC, MIST, and IACBaSTI include the solar-scaled models only.

Following Paper I, we use only the response curves of $G, G_{\mathrm{BP}}$, and $G_{\mathrm{RP}}$ from Gaia Collaboration et al. (2018b), but not from Weiler (2018), as well as $G$ photometry without a systematic correction proposed by Casagrande \& VandenBerg (2018, their equation (3)). However, this affects the fitting negligibly.

Following An et al. (2009), we adjust the SDSS model magnitudes using $\mathrm{AB}$ corrections given by Eisenstein et al. (2006): $u_{A B}=u-0.040, i_{A B}=i+0.015$, and $z_{A B}=z+0.030$, with no corrections in the $g$ and $r$ bands.

To derive the best reddening, distance, and age, we calculate the isochrones for a grid of some reasonable ages (1017 Gyr for MIST and IAC-BaSTI or 10-15 Gyr for the remaining models, with a step of $0.5 \mathrm{Gyr}$ ), distances (5.5-9.5 $\mathrm{kpc}$ with a step of $0.1 \mathrm{kpc}$ ) and reddenings [between -0.05

14 See Choi et al. (2018) for a discussion of an effect of varying mass loss and other parameters on CMDs. 
mag and the value calculated from $E(B-V)=0.06$ (the highest reasonable estimate) and the CCM89 extinction law with $R_{\mathrm{V}}=5$ (the highest reasonable value), with a step of 0.005 $\mathrm{mag}]$.

Each isochrone is represented by its authors as a discrete number of CMD points: this is seen in our figures with CMDs, especially for PARSEC with the most thinned-out isochrone points. Therefore, we select the best isochrone of each model as the one with a minimal total offset between the isochrone points and the fiducial points in the same magnitude range of the CMD. To pay more attention to CMD domains with more accurate photometry and well-defined models, we consider only fiducial points at the TO, SGB, $\mathrm{HB}$, the brighter part of the MS (higher than the magnitude limit applied in Sect. 3) and the parts of the RGB and AGB fainter than a CMD point where they meet.

As discussed in Paper I, the magnitudes of the HB and SGB better constrain the distance, the length and slope of the SGB and the magnitude difference between the HB and SGB - the age, while the overall colour offset of the isochrone w.r.t. the fiducial sequence - the reddening. This is taken into account for the balance of the uncertainties, presented in Appendix A.

\section{RESULTS}

As in Paper I, our results for different colours are consistent within their precision. Therefore, to avoid redundancy in this paper, we show some key examples of the CMDs with the best isochrone fits in Fig. 9, 10 and 11 (which can be compared with Fig. 1 and 2) as well as in Fig. B1 - B4 in Appendix B, while the ages, distances, and reddenings derived from some key isochrone fits are presented in Table 3. Figures and results for all the CMDs can be provided on request.

The predicted uncertainties of the derived distance, age and reddening are described in Appendix A. Also, the predicted uncertainties of the derived reddenings are given in the rightmost column 'Error' of Table 3. They can be compared with the maximal offsets of the isochrones w.r.t. the fiducial sequences along the reddening vector (i.e. nearly along the colour, or the abscissa). Such an offset is calculated for each isochrone in each CMD within the SGB, TO, brighter part of the MS, and fainter parts of the RGB and AGB, and presented in Table 3 after each value of reddening. The offset varies between 0.01 and $0.08 \mathrm{mag}$, with $0.03 \mathrm{mag}$ as a typical value, and can be accepted as the reddening precision. As is evident from Table 3, the offsets are comparable with the corresponding predicted uncertainties (the 'Error' column of Table 3) for the vast majority of isochrones and CMDs. This means that we take into account the uncertainties correctly. For each isochrone in each CMD, the largest value in the pair of the offset and the predicted uncertainty is adopted as the final accuracy of the derived reddening. It is used for the analysis in Sect. 5.1 and 6, as well as in Fig. 14 and 16.

The cases with a fiducial-isochrone offset $>0.08 \mathrm{mag}$ are marked in Table 3 as a fail fitting of the corresponding CMD. Also, we have to mark a model fail for a colour, if the best-fitting isochrone provides an improbable noticeable negative reddening [corresponding to $E(B-V)<-0.025$

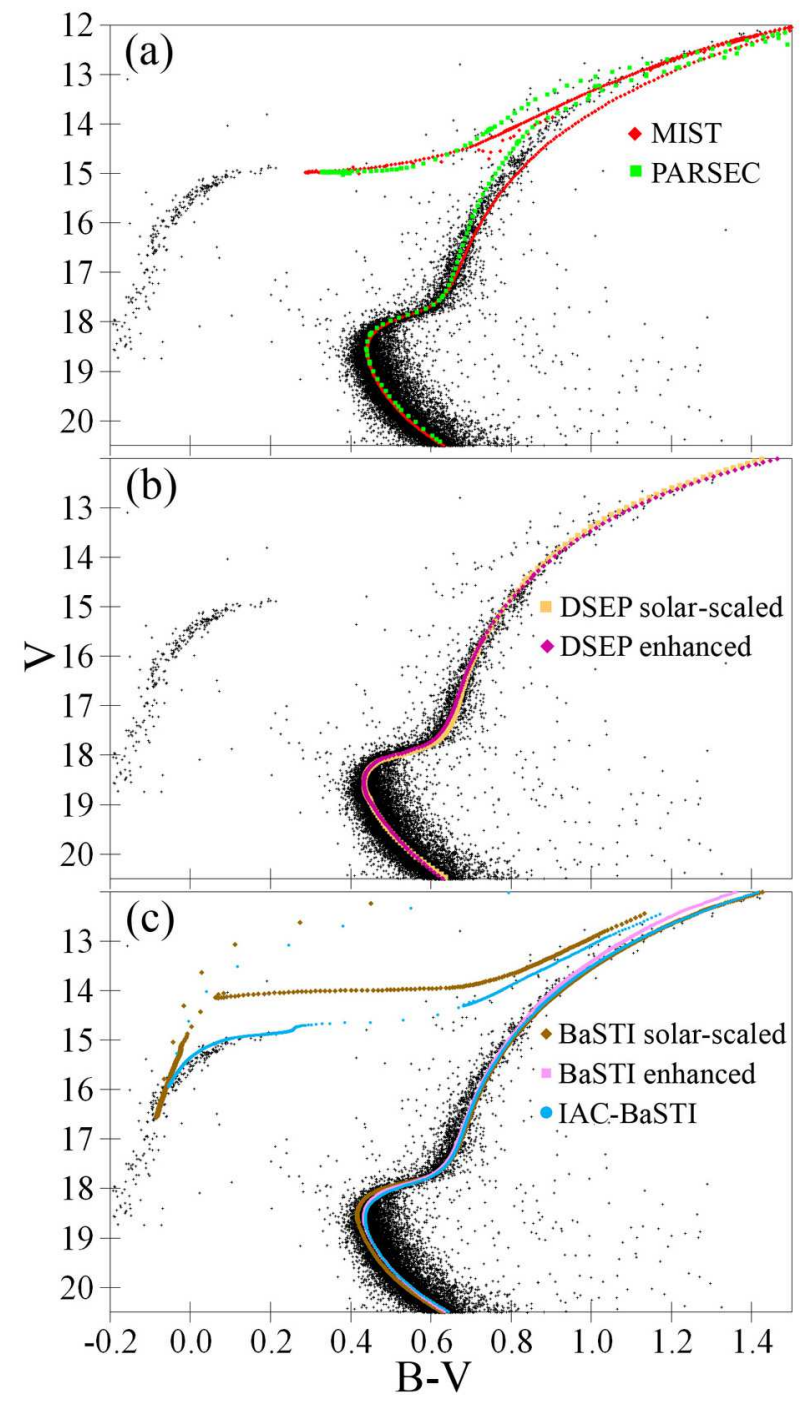

Figure 9. $B-V$ versus $V$ CMD of NGC 6205 based on the data from SPZ19 with the isochrones from solar-scaled PARSEC (green) and solar-scaled MIST (red) - upper plot, solar-scaled DSEP (yellow) and enhanced DSEP (magenta) - middle plot, solar-scaled BaSTI (brown), enhanced BaSTI (light purple), and solar-scaled IAC-BaSTI (blue) - lower plot, these are calculated with the best-fitting parameters from Table 3 .

mag]. However, a less noticeable negative or zero reddening is allowed for a CMD due to some errors of the data used or as a short-term feature of the observed extinction law. Fig. 11 presents some examples of such a fail fitting. In plot (a) the PARSEC isochrone for the distance $7800 \mathrm{pc}$, age $13 \mathrm{Gyr}$ and reddening $E(F 275 W-F 336 W)=0.021 \mathrm{mag}$ [about $E(B-V)=0.02 \mathrm{mag}$ ] fails to fit the main part of the RGB, providing too large an isochrone-to-data offset. A larger reddening would improve this fitting on the RGB, but at the expense of a worse fitting on the fainter RGB and MS. In plot (b) both the solar-scaled and enhanced DSEP isochrones for the zero reddening fail to fit the MS, TO and RGB, providing too large an isochrone-to-data offset. In this case, better fitting can be obtained, but at the expense of unreliable reddening $E(F 275 W-F 336 W)<-0.1 \mathrm{mag}$.

The age and distance to NGC 6205 are better deter- 
Table 3. The results of the isochrone fitting for various models and some key colours. To avoid confusion in the cases of the same colour, the source of photometry is indicated in brackets. The column 'Error' contains the predicted reddening uncertainty, which is calculated in Appendix A. In all the CMDs we use the colour as the abscissa and the magnitude in the redder filter as the ordinate: for example, $E(B-V)$ with the related age and distance is obtained by use of the $B-V$ versus $V$ CMD. The complete table is available online.

\begin{tabular}{|c|c|c|c|c|c|c|}
\hline & PARSEC & MIST & Solar-scaled DSEP & Enhanced DSEP & IAC-BaSTI & Error \\
\hline $\begin{array}{l}E(B-V)(\text { Rey et al. 2001) } \\
\text { age, Gyr } \\
\text { distance, pc }\end{array}$ & $\begin{array}{c}0.044 \pm 0.03 \\
12.5 \\
7600\end{array}$ & $\begin{array}{c}0.055 \pm 0.03 \\
14.5 \\
7400\end{array}$ & $\begin{array}{c}0.017 \pm 0.02 \\
14.5 \\
7700\end{array}$ & $\begin{array}{c}0.045 \pm 0.02 \\
12.5 \\
7200\end{array}$ & $\begin{array}{c}0.013 \pm 0.02 \\
15.0 \\
7500\end{array}$ & 0.027 \\
\hline $\begin{array}{l}E(B-V)(\mathrm{SPZ} 19) \\
\text { age, Gyr } \\
\text { distance, pc }\end{array}$ & $\begin{array}{c}0.042 \pm 0.03 \\
13.0 \\
7400\end{array}$ & $\begin{array}{c}0.060 \pm 0.02 \\
15.0 \\
7200\end{array}$ & $\begin{array}{c}0.018 \pm 0.02 \\
15.0 \\
7300\end{array}$ & $\begin{array}{c}0.045 \pm 0.02 \\
12.5 \\
7200\end{array}$ & $\begin{array}{c}0.020 \pm 0.02 \\
14.5 \\
7500\end{array}$ & 0.032 \\
\hline $\begin{array}{l}E(V-I)(\mathrm{SPZ} 19) \\
\text { age, Gyr } \\
\text { distance, pc }\end{array}$ & $\begin{array}{c}0.018 \pm 0.02 \\
13.5 \\
7700\end{array}$ & $\begin{array}{c}0.046 \pm 0.02 \\
14.5 \\
7600\end{array}$ & $\begin{array}{c}0.026 \pm 0.03 \\
15.0 \\
7400\end{array}$ & $\begin{array}{c}0.050 \pm 0.02 \\
12.5 \\
7200\end{array}$ & $\begin{array}{c}0.017 \pm 0.02 \\
14.0 \\
7800\end{array}$ & 0.033 \\
\hline $\begin{array}{l}E(I-W 1)(\mathrm{SPZ} 19) \\
\text { age, Gyr } \\
\text { distance, pc }\end{array}$ & $\begin{array}{c}0.065 \pm 0.02 \\
11.5 \\
8100\end{array}$ & $\begin{array}{c}0.081 \pm 0.03 \\
14.0 \\
8000\end{array}$ & $\begin{array}{c}0.073 \pm 0.03 \\
13.5 \\
7600\end{array}$ & $\begin{array}{c}0.076 \pm 0.03 \\
12.0 \\
7400\end{array}$ & $\begin{array}{c}0.089 \pm 0.03 \\
13.5 \\
7900\end{array}$ & 0.038 \\
\hline $\begin{array}{l}E\left(G_{\mathrm{BP}}-G_{\mathrm{RP}}\right) \\
\text { age, Gyr } \\
\text { distance, pc }\end{array}$ & $\begin{array}{c}0.044 \pm 0.03 \\
12.5 \\
7800\end{array}$ & $\begin{array}{c}0.046 \pm 0.03 \\
14.0 \\
7600\end{array}$ & $\begin{array}{c}0.053 \pm 0.02 \\
13.5 \\
7600\end{array}$ & $\begin{array}{c}0.066 \pm 0.02 \\
12.0 \\
7200\end{array}$ & $\begin{array}{c}0.020 \pm 0.03 \\
14.5 \\
7400\end{array}$ & 0.034 \\
\hline $\begin{array}{l}E\left(G_{\mathrm{RP}}-W 1\right) \\
\text { age, Gyr } \\
\text { distance, pc }\end{array}$ & $\begin{array}{c}0.089 \pm 0.02 \\
11.5 \\
7900\end{array}$ & $\begin{array}{c}0.074 \pm 0.03 \\
13.5 \\
7700\end{array}$ & $\begin{array}{c}0.092 \pm 0.02 \\
14.0 \\
7500\end{array}$ & $\begin{array}{c}0.120 \pm 0.02 \\
12.5 \\
7400\end{array}$ & $\begin{array}{c}0.083 \pm 0.02 \\
12.5 \\
8100\end{array}$ & 0.034 \\
\hline $\begin{array}{l}E(b-y)(\text { GVA98) } \\
\text { age, Gyr } \\
\text { distance, pc }\end{array}$ & $\begin{array}{c}0.008 \pm 0.04 \\
13.5 \\
7600\end{array}$ & $\begin{array}{c}0.035 \pm 0.03 \\
14.5 \\
7200\end{array}$ & $\begin{array}{c}0.035 \pm 0.03 \\
14.0 \\
7000\end{array}$ & $\begin{array}{c}0.049 \pm 0.01 \\
11.5 \\
7000\end{array}$ & $\begin{array}{c}0.020 \pm 0.02 \\
14.0 \\
7300\end{array}$ & 0.039 \\
\hline $\begin{array}{l}E(y-W 1)(\mathrm{SMB} 18) \\
\text { age, Gyr } \\
\text { distance, pc }\end{array}$ & $\begin{array}{c}0.132 \pm 0.02 \\
12.0 \\
7700\end{array}$ & $\begin{array}{c}0.123 \pm 0.03 \\
14.0 \\
7700\end{array}$ & $\begin{array}{c}0.102 \pm 0.03 \\
13.5 \\
7500\end{array}$ & $\begin{array}{c}0.126 \pm 0.02 \\
12.5 \\
7300\end{array}$ & $\begin{array}{c}0.102 \pm 0.03 \\
13.0 \\
7900\end{array}$ & 0.039 \\
\hline $\begin{array}{l}E(V-K)(\mathrm{BSV} 10) \\
\text { age, Gyr } \\
\text { distance, pc }\end{array}$ & $\begin{array}{c}0.094 \pm 0.03 \\
13.0 \\
7200\end{array}$ & $\begin{array}{c}0.110 \pm 0.03 \\
15.5 \\
6900\end{array}$ & $\begin{array}{c}0.083 \pm 0.03 \\
14.0 \\
7200\end{array}$ & $\begin{array}{c}0.110 \pm 0.03 \\
13.0 \\
6900\end{array}$ & $\begin{array}{c}0.088 \pm 0.03 \\
14.5 \\
7200\end{array}$ & 0.034 \\
\hline
\end{tabular}

Table 4. The mean and median age (Gyr) and distance (pc) derived for the various models.

\begin{tabular}{|c|c|c|c|c|c|c|c|}
\hline & \multicolumn{7}{|c|}{ Model } \\
\hline & PARSEC & MIST & $\begin{array}{c}\text { DSEP } \\
\text { Solar-scaled }\end{array}$ & $\begin{array}{c}\text { DSEP } \\
\text { Enhanced }\end{array}$ & $\begin{array}{c}\text { BaSTI } \\
\text { Solar-scaled }\end{array}$ & $\begin{array}{c}\text { BaSTI } \\
\text { Enhanced }\end{array}$ & IAC-BaSTI \\
\hline Mean distance & $7630 \pm 316$ & $7430 \pm 474$ & $7520 \pm 368$ & $7220 \pm 272$ & $7100 \pm 250$ & $6844 \pm 260$ & $7462 \pm 359$ \\
\hline Median distance & 7700 & 7400 & 7500 & 7200 & 7100 & 6900 & 7400 \\
\hline Mean distance for $0.45<\lambda<0.9 \mathrm{~nm}$ & $7545 \pm 267$ & $7289 \pm 264$ & $7416 \pm 279$ & $7147 \pm 244$ & $7125 \pm 255$ & $6814 \pm 212$ & $7314 \pm 280$ \\
\hline Median distance for $0.45<\lambda<0.9 \mathrm{~nm}$ & 7600 & 7300 & 7500 & 7200 & 7100 & 6900 & 7350 \\
\hline Mean age & $12.6 \pm 0.9$ & $14.3 \pm 0.8$ & $13.8 \pm 1.2$ & $12.1 \pm 0.8$ & $14.8 \pm 0.5$ & $14.6 \pm 0.1$ & $14.1 \pm 0.7$ \\
\hline Median age & 12.5 & 14.0 & 14.0 & 12.5 & 15.0 & 15.0 & 14.0 \\
\hline Mean age for $0.45<\lambda<0.9 \mathrm{~nm}$ & $12.8 \pm 0.8$ & $14.4 \pm 0.7$ & $14.4 \pm 0.6$ & $12.3 \pm 0.7$ & $15.0 \pm 0.0$ & $14.9 \pm 0.2$ & $14.3 \pm 0.3$ \\
\hline Median age for $0.45<\lambda<0.9 \mathrm{~nm}$ & 13.0 & 14.5 & 14.5 & 12.5 & 15.0 & 15.0 & 14.5 \\
\hline
\end{tabular}



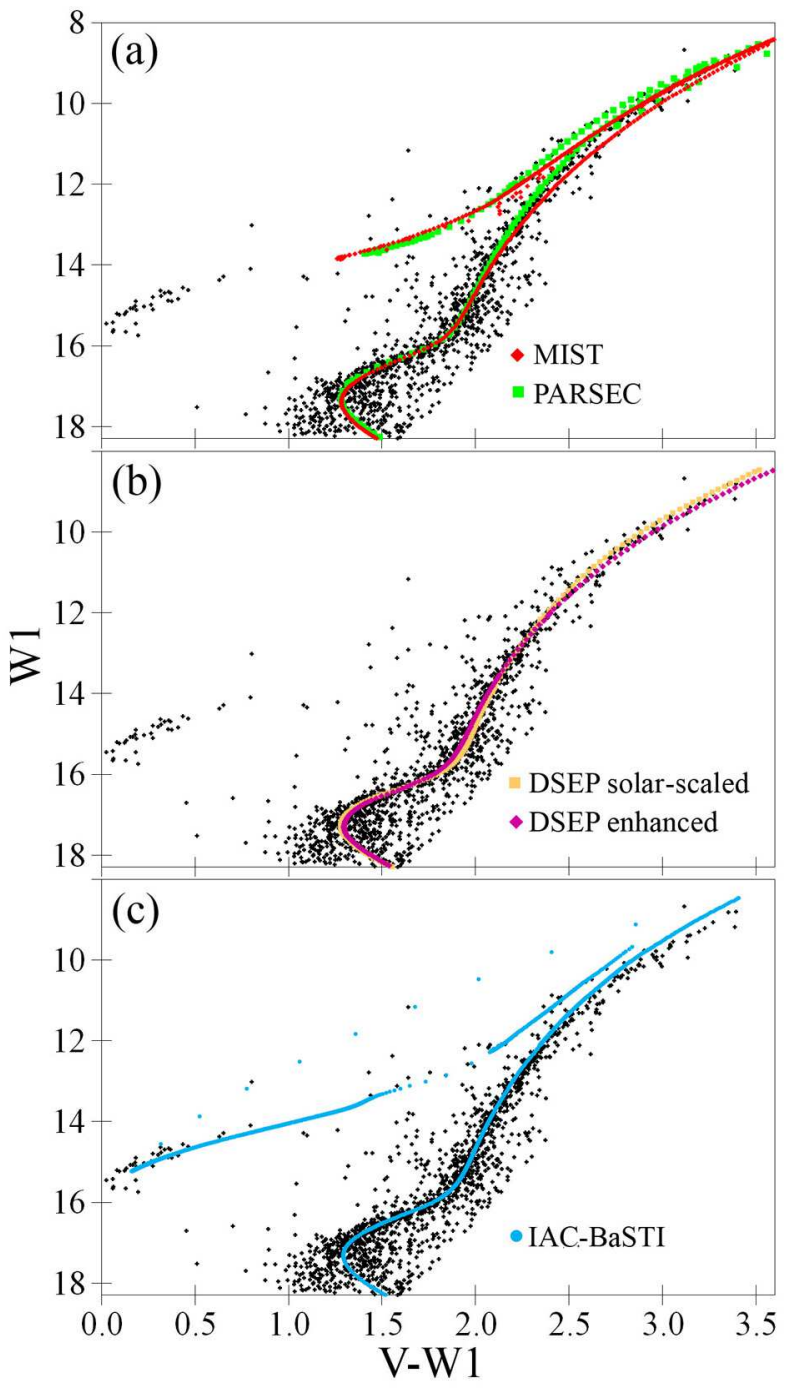

Figure 10. The same as Fig. 9 but for $V-W 1$ versus $W 1$ based on the data from SPZ19 and unWISE.

mined by use of the models and data for both the HB and SGB (PARSEC, MIST and IAC-BaSTI), while DSEP and BaSTI allow us to consider the difference between the solarscaled and enhanced isochrones. However, BaSTI gives few isochrones (as seen in the online version of Table 3), with no IR band, as well as unreliable ages about 15 Gyr (even older, if BaSTI was not limited to $15 \mathrm{Gyr}$ ).

Table 4 presents the mean and median estimates as well as standard deviations ${ }^{15}$ of age and distance from the models. These values appear different for the UV, optical $(0.45<\lambda<0.9 \mathrm{~nm})$ and IR ranges ${ }^{16}$. This is evident from Fig. 12, where the distances and ages are presented as functions of $1 / \lambda^{17}$. This issue must be investigated in future. Assuming that the models and isochrones might be more

15 The standard deviation \pm 0.0 for solar-scaled BaSTI means that all the estimates are 15 Gyr.

16 Here we calculate $1 / \lambda$ for the colour under consideration as an average of $1 / \lambda_{\text {eff }}$ for the filters used, where $\lambda_{\text {eff }}$ is taken from Table 1.

17 The solar-scaled DSEP isochrones can be calculated only to
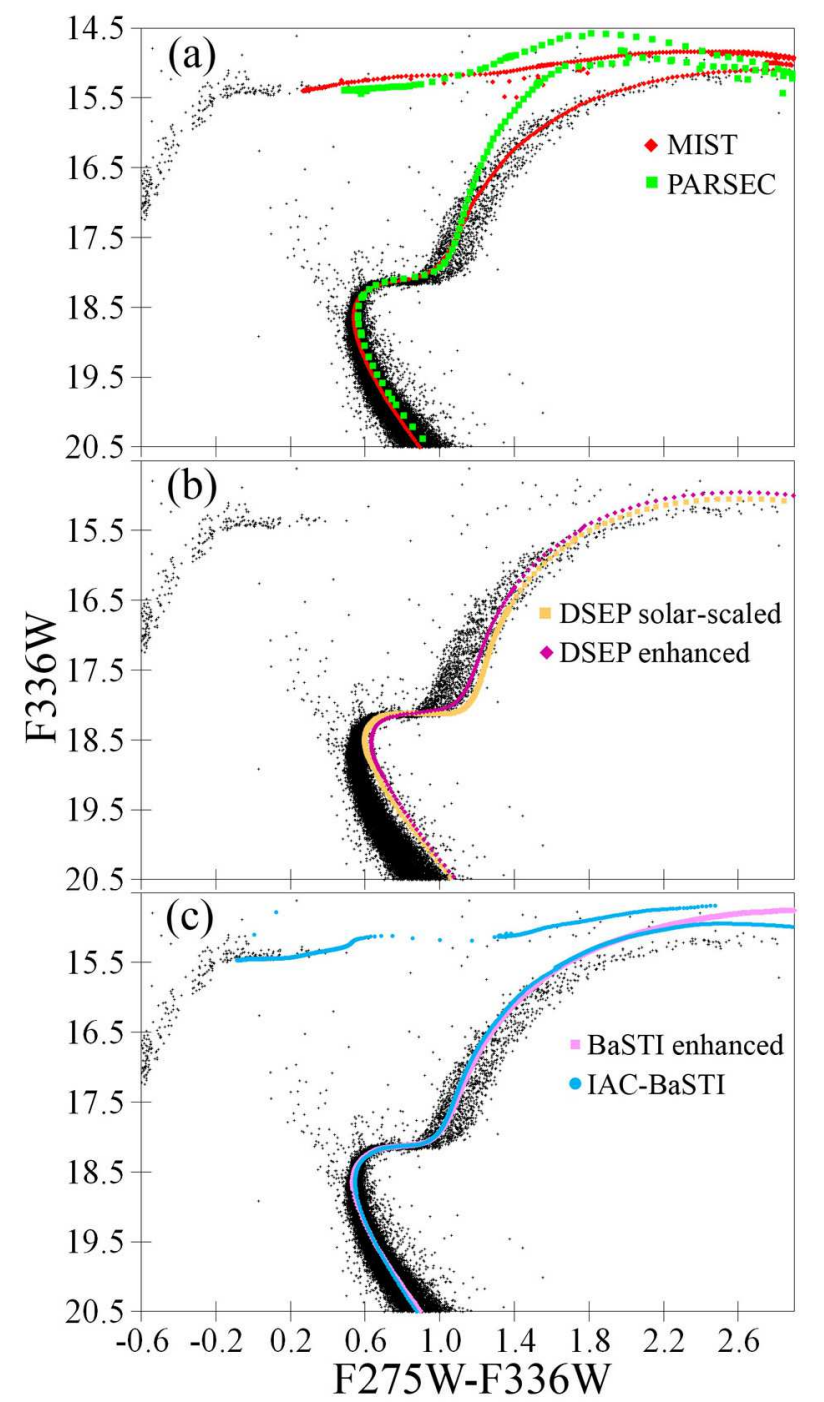

Figure 11. The same as Fig. 9 but for $F 275 W-F 336 W$ versus F336W based on the HST/WFC3 data.

reliable in the optical range, we use only this range in order to calculate the most probable estimates of the distance and age.

The different datasets with one model applied provide rather consistent distances and ages, at least, in the optical range, as is evident from Fig. 12 and from the standard deviations in Table 4 . The predicted uncertainties of the distance and age $( \pm 340 \mathrm{pc}$ and \pm 1 Gyr, respectively) from Appendix A agree with the standard deviations in Table 4 within the optical range and slightly underestimate the deviations within the full wavelength range.

However, the distance and age estimates from Table 4 show a considerable systematic discrepancy for the different models, which exceeds the predicted uncertainties. This discrepancy exists in the optical range as well. This follows the high diversity of NGC 6205 estimates, mentioned in Sect. 2, and the note for age by Saracino et al. (2019): 'Differences in

15 Gyr, thus, providing only the lower estimate for some of its results, as seen from Fig. 12. 


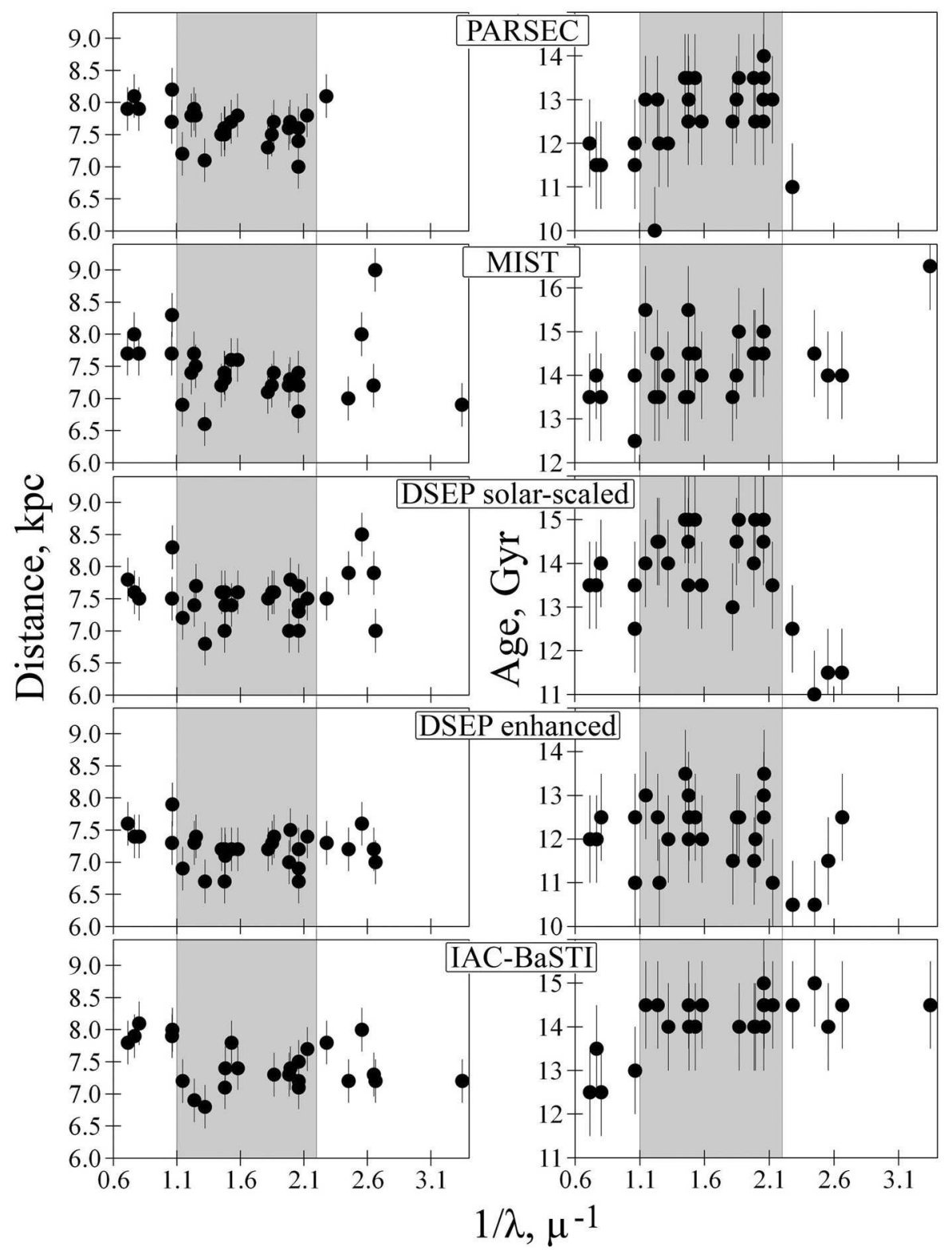

Figure 12. The distances and ages, derived by use of the different models, as functions of $1 / \lambda$. The error bars are of 340 pc and 1 Gyr, respectively. The grey area is the wavelength range within $0.45<\lambda<0.9 \mathrm{~nm}$.

the best-fit age are somewhat expected when different models, which adopt slightly different solar abundances, opacities, reaction rates, efficiency of atomic diffusion etc., are compared'.

Fig. 13 presents a diagram of effective temperature $T_{\text {eff }}$ versus luminosity $L$ (w.r.t. the solar one) with the different isochrones. These isochrones are calculated for the bestfitting median ages taken from Table 4 for the optical range. A reasonable variation of these ages provides a negligible shift of these isochrones and does not change our conclusions. Such a diagram is independent of distance, reddening, wavelength range, colour- $T_{\text {eff }}$ relations and bolometric corrections used. Hence, it is a direct comparison of the models.

Fig. 13 explains the bulk of the systematic differences between the estimates from different models in Table 4. For example, PARSEC gives the youngest age due to the short- est SGB, while both solar-scaled and enhanced BaSTI give the oldest ages due to the longest SGB. IAC-BaSTI gives an age older than PARSEC does due to a larger HB-SGB luminosity difference. Although this difference for MIST is also small, its long SGB makes the MIST ages rather old. The derived distances in Table 4 also follow the luminosity of the SGB and TO in Fig. 13 for all the models: from the shortest distance and faintest SGB/TO for the enhanced BaSTI to the longest distance and brightest SGB/TO for PARSEC.

Extinction and reddening, provided by an isochrone, is primarily defined by an overall (or median) $T_{\text {eff }}$ of this isochrone. Fig. 13 shows that IAC-BaSTI gives the reddest isochrone and, consequently, needs the lowest reddening to fit such an isochrone with data. BaSTI and solarscaled DSEP give slightly bluer isochrones, followed by PARSEC, MIST and, finally, by enhanced DSEP with the bluest 


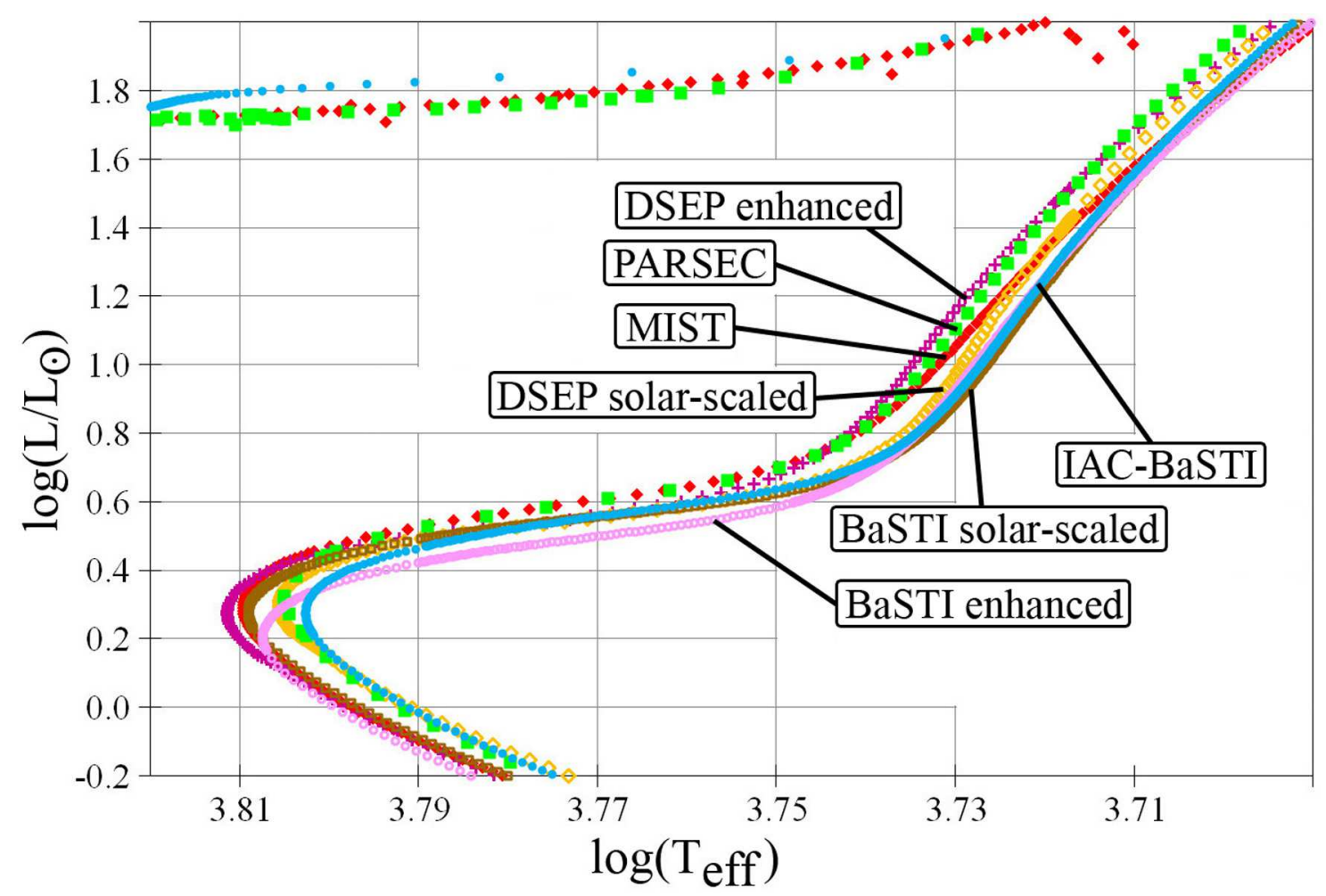

Figure 13. $\log \left(T_{\text {eff }}\right)$ versus $\log (L / L \odot)$ diagram with the isochrones from PARSEC (green squares), MIST (red diamonds), solar-scaled DSEP (yellow open diamonds), enhanced DSEP (magenta crosses), solar-scaled BaSTI (brown open squares), enhanced BaSTI (light purple circles), and solar-scaled IAC-BaSTI (blue circles) for the best-fitting median ages for $0.45<\lambda<0.9 \mathrm{~nm}$ taken from Table 4 . This figure shows only the stages of stellar evolution used in the isochrone-to-data fitting.

isochrone. This explains the lowest extinction/reddening estimates from IAC-BaSTI, higher ones from PARSEC, even higher ones from MIST and the highest ones from the enhanced DSEP in Sect. 5.1 and 6.

Thus, the diversity of the distance, age, extinction and reddening estimates from the different models is mainly due to some differences of the models before the colour $-T_{\text {eff }}$ relations and bolometric corrections are applied.

In Fig. 9 - 11 and other CMDs, BaSTI and DSEP show quite little (a few hundredths of a magnitude) difference between the best-fitting solar-scaled and enhanced isochrones. However, Table 3 and 4 show that the derived age, reddening and distance, for the best-fitting solar-scaled and enhanced isochrones in the same CMD, differ considerably.

Fig. 13 shows that the enhancement makes both the DSEP and BaSTI isochrones fainter, which is followed by a shorter distance for the enhanced models. However, the enhancement decreases the age both for DSEP and BaSTI, despite a negligible change of the length of the SGB, as seen from Fig. 13. Therefore, this age decrease may be due to the colour $-T_{\text {eff }}$ relations and/or bolometric corrections. Also, Fig. 13 shows that the enhanced DSEP isochrone is much bluer than the solar-scaled one, while there is no such a difference for BaSTI. Thus, the effect of enhancement on the derived parameters is not clear.

We decide to use only PARSEC, MIST, enhanced DSEP and IAC-BaSTI median estimates for $0.45<\lambda<0.9 \mathrm{~nm}$ in the calculation of the most probable age and distance.

The most probable distance for NGC 6205 is $7.4 \pm 0.2$ $\mathrm{kpc}$, as a middle value for the interval $7.2-7.6 \mathrm{kpc}$ of the PARSEC, MIST, enhanced DSEP and IAC-BaSTI estimates. The uncertainty includes both random and systematic uncertainties. The correspondent true distance modulus is $(m-M)_{0}=14.35 \pm 0.06 \mathrm{mag}$, while the parallax is $0.135 \pm 0.004$ mas. This agrees within the uncertainties with recent estimate of the parallax from Gaia DR2 $0.0801+0.029=0.1091 \pm 0.040$ mas, where 0.029 and 0.040 mas are the parallax global zero-point correction derived from the analysis of the high-precision quasar sample and the median uncertainty in parallax, respectively (Lindegren et al. 2018). This Gaia DR2 parallax corresponds to the distance $R=9.2_{-2.5}^{+5.3} \mathrm{kpc}$.

The age of NGC 6205 remains quite uncertain until the development of some new enhanced models. We assume with caution that the most probable age for NGC 6205 is 13.5 Gyr, as the middle value for the interval 12.5-14.5 Gyr of the PARSEC, MIST, enhanced DSEP and IAC-BaSTI estimates, with an uncertainty of 1 Gyr covering the whole interval of these estimates.

In Paper I we have shown with similar data and using a similar approach that any reasonable variation of metallicity, abundance, age and distance cannot change an isochrone colour. Hence, such a colour w.r.t. a fiducial sequence provides us with an estimate of the reddening. Each pair of a model and a dataset provides its own set of reddenings for different wavelengths and, consequently, its own extinction law. Below, we compare them with each other and with the most popular extinction laws. 


\subsection{Preliminary extinction law}

Table 3 shows that SPZ19 and Rey et al. (2001), in combination with PARSEC, MIST, enhanced DSEP and IACBaSTI, give an agreed direct estimate of the average $E(B-$ $V)=0.04 \mathrm{mag}$ with both the random and systematic uncertainty $0.01 \mathrm{mag}$, assuming that systematic errors of the different models/isochrones compensate each other.

PARSEC, MIST, DSEP and IAC-BaSTI, in contrast to BaSTI, provide us with IR colours. As discussed in Paper I, the IR extinction and its variation due to some reasonable variations of the extinction law are very low. For example, $E(B-V)=0.04 \mathrm{mag}$ from our results with the CCM89 extinction law with its reasonable variations within $2.6<R_{\mathrm{V}}<3.6^{18}$ corresponds to $0.011<A_{\mathrm{K}}<0.018$ and $0.006<A_{\mathrm{W} 1}<0.009$ mag. Such estimates would be two times less with $E(B-V)=0.02$ from Harris (1996). Hence, we can initially adopt $A_{\mathrm{K}}=0.012 \pm 0.006$ and $A_{\mathrm{W} 1}=0.006 \pm 0.003$ mag, where both the uncertainties of the extinction and extinction law are included. Thus, the uncertainty of the IR extinction is several thousandths of a magnitude. This is a minor contribution into the balance of the uncertainties discussed in Appendix A.

We calculate the extinction in the remaining 32 bands, by use of these IR extinctions calculated with $R_{\mathrm{V}}=3.1$, in combination with the reddenings derived from the isochrone fitting. In such an approach, we have to adopt an extinction law only for the first iteration, i.e. to calculate $A_{\mathrm{K}}$ and $A_{\mathrm{W} 1}$. Then we derive $R_{\mathrm{V}}$ from the extinctions in the remaining bands. In the next iteration we recalculate $A_{\mathrm{K}}$ and $A_{\mathrm{W} 1}$ by use of the CCM89 extinction law with the derived $R_{\mathrm{V}}$. Since $R_{\mathrm{V}} \approx 3.1$ for NGC 6205 , as shown later, we need only a few iterations to converge. The derived $A_{\mathrm{K}} \approx 0.014$ and $A_{\mathrm{W} 1} \approx 0.007$ (slightly different for the different models) define the IR extinction zero-points for the datasets with IR observations, as mentioned in Sect. 3.

Our reddening results do not contradict previous results by other authors on a short wavelength baseline. For example, the average $E(b-y)=0.028 \pm 0.018 \mathrm{mag}$ from PARSEC, MIST, enhanced DSEP and IAC-BaSTI in Table 3 does not contradict the estimate $E(b-y)=0.015 \pm 0.010$ by GVA98 for the same data but using a different method, however, our estimate is almost twice as high. However, a longer wavelength baseline provides us with a more precise reddening/extinction estimate and also smoothes possible short-term variations of the extinction law.

The reddening $E(B-V)$ represents the extinction law only on a short wavelength baseline, while the extinction $A_{\mathrm{V}}$ is well defined on a much longer baseline as

$A_{\mathrm{V}} \approx A_{\mathrm{V}}-A_{\mathrm{W} 1}=E(V-W 1)$.

Therefore, given the estimates for $A_{\mathrm{K}}$ and $A_{\mathrm{W} 1}$, we calculate $A_{\mathrm{V}}$ from equation (2), or from similar equations with $K$ instead of $W 1$ or $y$ instead of $V$, for each model by averaging the isochrone fitting results for the datasets of (i) SPZ19, (ii) BSV10, (iii) SMB18 and GVA98. We obtain preliminary $A_{\mathrm{V}}=0.112,0.131,0.132$, and $0.106 \mathrm{mag}$ for PARSEC, MIST, enhanced DSEP, and IAC-BaSTI, respectively, which will be adjusted in Sect. 6. The uncertainty of these values can be

18 CCM89 do not provide $A_{\mathrm{W} 1}$, but it is extrapolated by us from the CCM89 IR extinction law. estimated as $\pm 0.02 \mathrm{mag}$. The average of these estimates is $A_{\mathrm{V}}=0.120 \pm 0.014 \mathrm{mag}$.

Given $A_{\mathrm{V}}=0.120 \pm 0.014$ and $E(B-V)=0.04 \pm 0.01 \mathrm{mag}$, we obtain $R_{\mathrm{V}}=3.0_{-0.9}^{+1.5}$. Since this value is quite uncertain and is not significantly different from the value $R_{\mathrm{V}}=3.1$, we decide to keep the latter in our further calculations.

Fig. 14 shows the empirical extinction laws derived from the isochrone fitting for different models and datasets. The different datasets are shown by different colours. The uncertainties of the extinctions are calculated from the uncertainties of the reddenings and shown in Fig. 14 by the vertical bars. Fig. 14 shows a moderate agreement between the empirical extinction laws from the different models and datasets: a smooth extinction law curve can be drawn through all the results within their uncertainty bars. However, some noticeable offsets are seen for some datasets, such as the SDSS and Gaia ones.

The isochrone fitting provides us with the reddenings, which are seen in Fig. 14 as the relative vertical positions of the points. Hence, our result is an overall tilt of the bulk of the points in Fig. 14. Therefore, given the fitting results, we cannot shift vertically the points w.r.t. each other. Also, we cannot shift down the bulk of the points, as a whole, due to a non-negative $A_{\mathrm{W} 1}$.

The thick grey curve in Fig. 14 shows the extinction law of Schlafly et al. (2016) with $E(B-V)=$ $0.015 \pm 0.001$ and $A_{\mathrm{V}}=0.044 \pm 0.003$ taken from Schlafly \& Finkbeiner (2011) [recalibrated 2D reddening map of Schlegel, Finkbeiner \& Davis (1998, hereafter SFD) with $E(B-V)=0.017$ and $\left.A_{\mathrm{V}}=0.051 \mathrm{mag}\right]{ }^{19}$. We do not draw other popular extinction laws, such as, those of CCM89, Fitzpatrick (1999) and Wang \& Chen (2019) with the same low $A_{\mathrm{V}}$, since they would be indistinguishable from the law of Schlafly et al. (2016) in the scale of Fig. 14, except for their slight deviation in the UV. However, using three black curves we depict the CCM89 extinction law with the derived high $A_{\mathrm{V}}$ and different $R_{\mathrm{V}}$.

It is seen from Fig. 14 that the grey curve of Schlafly et al. (2016)'s extinction law is very far from our results and from all three curves of the CCM89 extinction law. The proximity of the three CCM89 extinction laws with each other means that $R_{\mathrm{V}}$ does not matter and can be determined from our data with a low accuracy only. In contrast, the deviation of the CCM89 extinction laws with the high $A_{\mathrm{V}}$ from Schlafly et al. (2016)'s extinction law with the low $A_{\mathrm{V}}$ means that $A_{\mathrm{V}}$ does matter and can be determined from these data fairly accurately.

We emphasize the key inconsistency between our average estimate $A_{\mathrm{V}}=0.120 \pm 0.014 \mathrm{mag}$ (or even our lowest estimate $A_{\mathrm{V}}=0.106 \pm 0.020 \mathrm{mag}$ from IAC-BaSTI) and that of Schlafly \& Finkbeiner $(2011), A_{\mathrm{V}}=0.044 \pm 0.003$ mag, both determined on some long wavelength baselines. Such long baselines guarantee that this inconsistency cannot be resolved by a reasonable variation of the extinction law. Thus, all the datasets and models presented in Fig. 14 show that $A_{\mathrm{V}}$ from Schlafly \& Finkbeiner (2011) and, consequently, from SFD as the original source, may be underes-

19 Also Schlafly \& Finkbeiner (2011) find a negligible differential reddening in the field of NGC 6205 within $0.012<E(B-V)<0.017$ mag. 


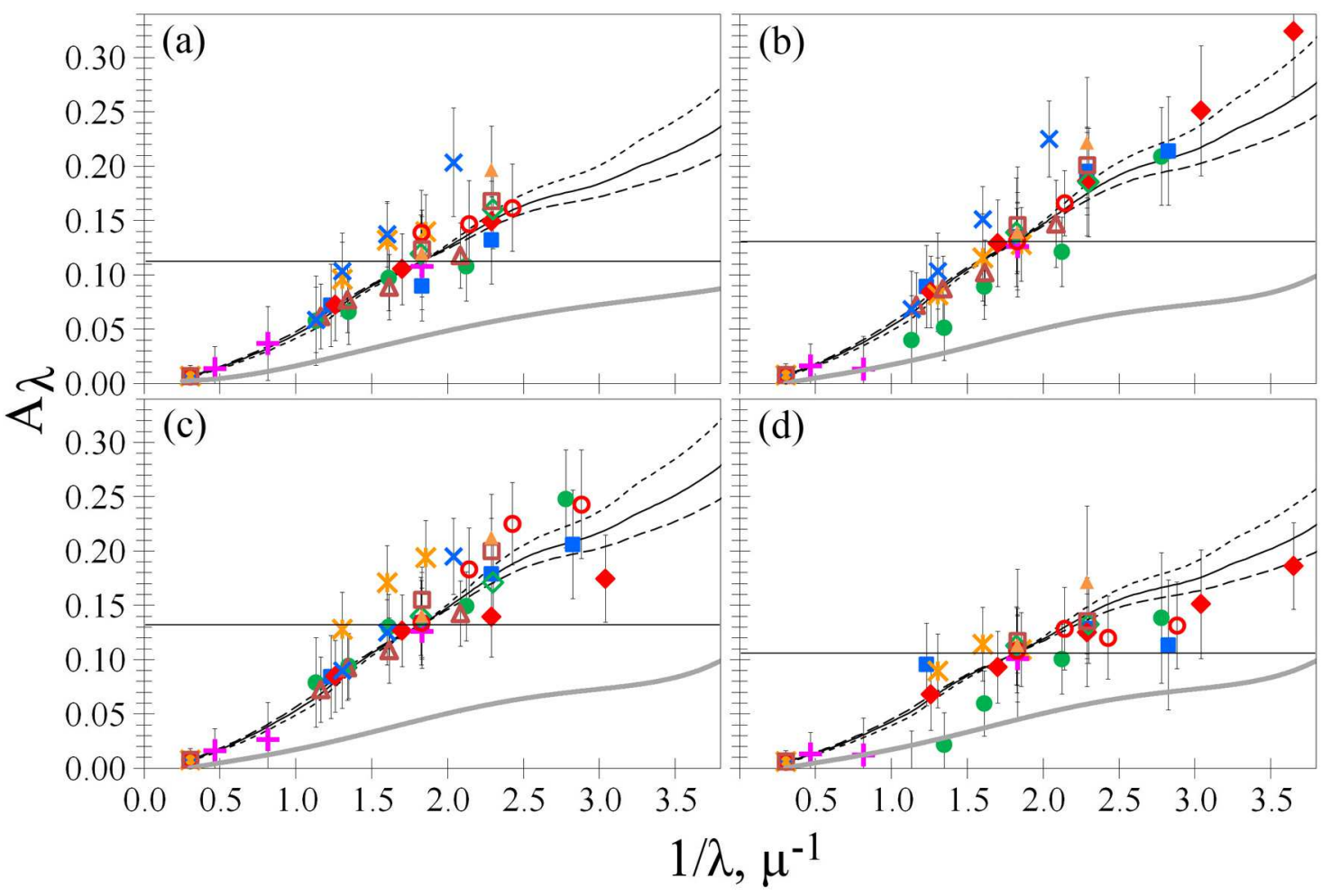

Figure 14. The empirical extinction laws from the isochrone fitting by (a) PARSEC, (b) MIST, (c) enhanced DSEP, (d) IAC-BaSTI. The datasets with an IR extinction zero-point: $U B V I$ by SPZ19 - blue squares, BV by Rey et al. (2001) - open brown squares, Gaia - yellow snowflakes, SDSS - green circles, Strömgren by SMB18 and GVA98 - open red circles, VJK by BSV10 - purple upright crosses, and the datasets with a zero-point defined by the CCM89 extinction law with $R_{\mathrm{V}}=3.1:$ HST ACS/WFC3 - red diamonds, HST WFPC2 - open green diamonds, $B V$ by PFF98 - yellow triangles, Pan-STARRS - open brown triangles, CFHT/MegaCam by Clem, VandenBerg \& Stetson (2008) - blue inclined crosses. The black dotted, solid and dashed curves show the CCM89 extinction law with $R_{\mathrm{V}}=2.6,3.1$, and 3.6, respectively, and with the derived $A_{\mathrm{V}}$, which is shown by the horizontal line. The thick grey curve shows the extinction law of Schlafly et al. (2016) with $A_{\mathrm{V}}=0.044 \mathrm{mag}$.

timated. Our results seem to be more reliable, since they are based on the multiband photometry on a long wavelength baseline, while the SFD reddening estimate is based on some measurements of IR emission and emission-to-reddening calibration. Hence, the possible underestimation of the reddening by SFD may be due to a wrong emission-to-reddening calibration of low reddenings in SFD, as discussed, for example, by Gontcharov \& Mosenkov (2018).

\section{ADJUSTMENT}

Some inherent offsets of the model colours, being different for different datasets, may explain the vertical offsets (i.e. the different extinction laws) of the datasets, which are seen in Fig. 14.

Similar to Paper I, to verify this assumption, we analyse some CMDs obtained with pairs of filters from different datasets but of similar effective wavelengths. Such filters must have a difference of $<0.2$ in their extinction coefficients. In such a case, any reasonable variation of the reddening [e.g. within $0.01<E(B-V)<0.06 \mathrm{mag}$ ] cannot shift any isochrone in such a CMD along the colour by more than $0.2 \times(0.06-0.01)=0.01 \mathrm{mag}$. This is smaller than the colour offsets under consideration. Any reasonable variation of $[\mathrm{Fe} / \mathrm{H}]$, abundance, age, and distance also cannot change these isochrone colours by more than $0.01 \mathrm{mag}$. This allows us to consider any colour offset between an isochrone and the fiducial sequence in such a CMD as a systematic error (offset) of this isochrone.

Four such CMDs with isochrones from PARSEC, MIST, enhanced DSEP, ${ }^{20}$, and IAC-BaSTI for the age 10-15 Gyr, distance $7.4 \mathrm{kpc}, E(B-V)=0.04$ and CCM89 extinction law with $R_{V}=3.1$ are presented in Fig. 15. Also, we use the $F 438 W-B$ versus $B, G_{\mathrm{BP}}-y$ versus $y$, and $i_{\mathrm{SDSS}}-G_{\mathrm{BP}}$ versus $G_{\mathrm{BP}}$ CMDs, which are not shown in Fig. 15 . Thus, we only use the SDSS, Gaia DR2, SPZ19, HST, and Strömgren datasets.

In such a CMD, any fiducial sequence at the brightest and faintest magnitudes would be biased due to the sample incompleteness and larger photometric errors. In addition, the HB, AGB and RGB may be confused with each other, since the colours are close to zero. Therefore, we draw in Fig. 15 and use parts of the fiducial sequence for the fainter RGB, i.e. within a magnitude range between the HB and the SGB. It is seen that the scatter of the star colours in this magnitude range is a few hundredths of a magnitude, i.e. in a

20 The solar-scaled DSEP isochrones are not shown, since in all these CMDs and for all the ages they coincide within $0.01 \mathrm{mag}$ with the enhanced DSEP isochrones. 

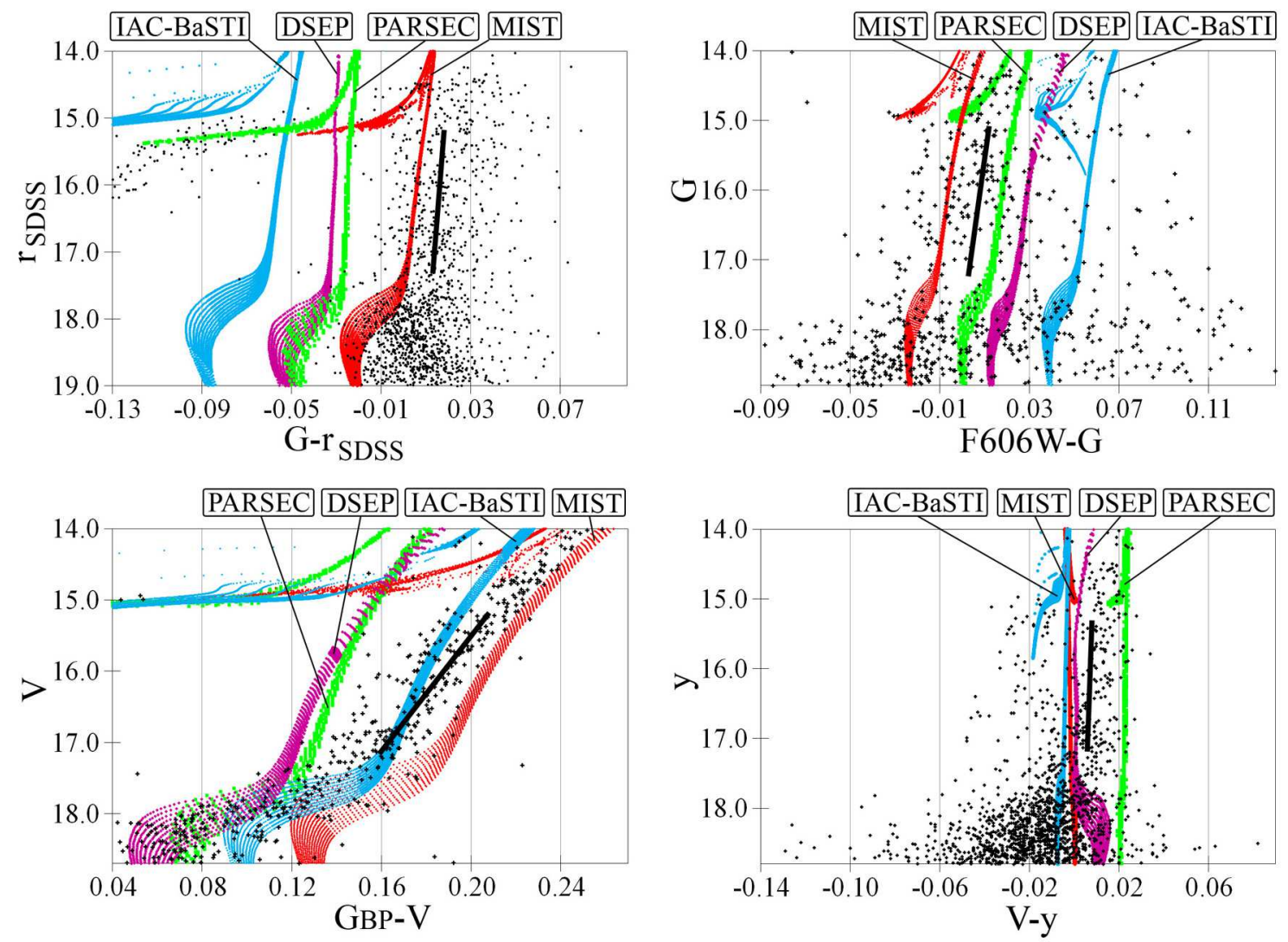

Figure 15. Some CMDs of NGC 6205 for pairs of filters from different datasets but of similar effective wavelengths. The individual stars are shown by the black dots. Part of the fiducial sequence for the RGB between the HB and the SGB is shown by the thick black line. The sets of isochrones for the ages from 10-15 Gyr, distance $7.4 \mathrm{kpc}$, reddening $E(B-V)=0.04$ mag and CCM89 extinction law with $R_{V}=3.1$ are shown by the dotted curves and coloured as follows: PARSEC - green; MIST - red; enhanced DSEP - magenta; and IAC-BaSTI - blue.

good agreement with the stated precision of the photometry. Moreover, it is evident from Fig. 15 that part of any fiducial sequence in this magnitude range must be very close to a straight line. Such parts of the fiducial sequences are shown in Fig. 15 by the thick black lines.

Also, it is evident from Fig. 15 that the colour offsets between these fiducial sequence lines and the parts of the isochrones within the same magnitude range can be derived with an accuracy better than 0.01 mag.

Fig. 15 shows that these colour offsets are different for different isochrones in the same CMD, as well as for different colours. This may be explained, among other reasons, by discordant colour $-T_{\text {eff }}$ relations and bolometric corrections used with each model for the different datasets, as discussed by Paxton et al. (2011); Choi et al. (2016); Hidalgo et al. (2018). Hence, these offsets can be used to simultaneously adjust the datasets: we search for some consistent colour corrections to shift each isochrone to the correspondent fiducial sequence line. This brings together the extinction estimates from a model for the different datasets, i.e. the datasets within each plot of Fig. 14. We adjust only the datasets, but not the models/isochrones w.r.t. each other, since we have no indication, which model/isochrone is better. Consequently, an additional condition of this adjustment is a minimal (nearly zero) absolute value of a sum of the corrections
Table 5. The offset corrections (mag) applied to the datasets and models.

\begin{tabular}{lrrrr}
\hline & \multicolumn{5}{c}{ Model } \\
Dataset & PARSEC & MIST & Enhanced DSEP & IAC-BaSTI \\
\hline SDSS & -0.02 & -0.02 & -0.01 & -0.04 \\
Gaia DR2 & 0.03 & -0.01 & 0.03 & 0.03 \\
SPZ19 & -0.01 & 0.02 & 0.00 & 0.02 \\
HST & 0.02 & 0.01 & -0.01 & -0.02 \\
Strömgren & -0.01 & 0.00 & -0.01 & 0.00 \\
\hline
\end{tabular}

to each model/isochrone. However, since only five datasets are used in this adjustment, we do not fix the average extinctions of the models, in contrast to Paper I. However, since these five datasets are the major ones, the average extinctions change slightly. Also, since we adjust only the colours, this does not change the derived ages and distances.

The resulting offset corrections applied to the datasets and models are shown in Table 5 . They demonstrate a consistency with each other and with the colour offsets in Fig. 15. This adjustment seems to reduce the systematic offsets of the used datasets to a level of $<0.01 \mathrm{mag}$. 


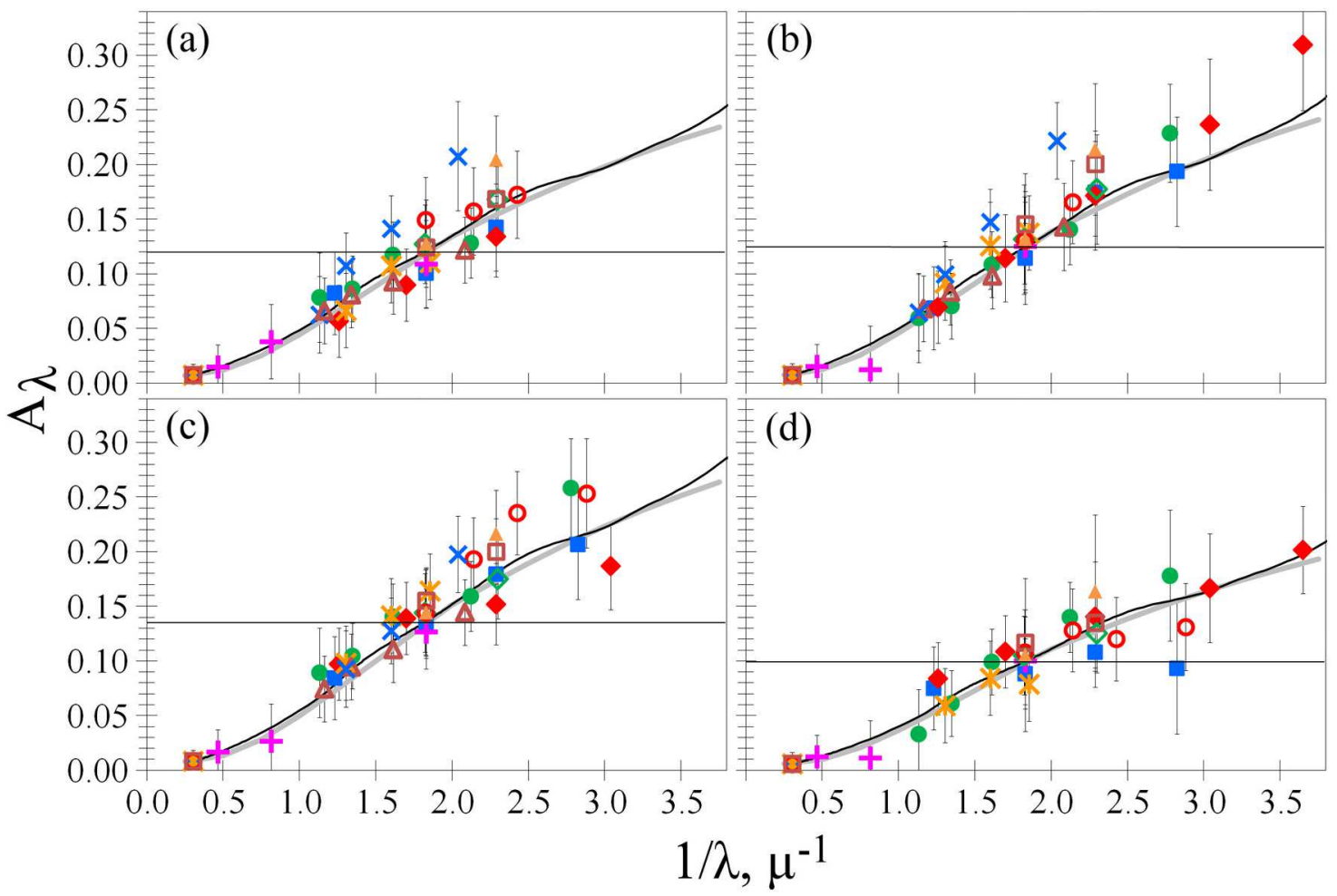

Figure 16. The empirical extinction laws from the isochrone fitting by (a) PARSEC, (b) MIST, (c) enhanced DSEP, (d) IAC-BaSTI after the adjustment for the corrections from Table 5. The datasets are: $U B V I$ by SPZ19 - blue squares, $B V$ by Rey et al. (2001) - open brown squares, Gaia - yellow snowflakes, SDSS - green circles, Strömgren by SMB18 and GVA98 - open red circles, VJK by BSV10 - purple upright crosses, HST ACS/WFC3 - red diamonds, HST WFPC2 - open green diamonds, BV by PFF98 - yellow triangles, Pan-STARRS - open brown triangles, CFHT/MegaCam by Clem, VandenBerg \& Stetson (2008) - blue inclined crosses. The datasets without an IR extinction zero-point have a zero-point defined by the CCM89 extinction law with $R_{\mathrm{V}}=3.1$. The thick grey and black solid curves show the extinction laws of Schlafly et al. (2016) and CCM89 with $R_{\mathrm{V}}=3.1$, respectively, with the derived $A_{\mathrm{V}}$, which is shown by the horizontal line.

Table 6. The estimates of $A_{\mathrm{V}}$ from the various datasets and models.

\begin{tabular}{lrrrrr}
\hline & \multicolumn{2}{c}{ Model } & & \\
Dataset & PARSEC & MIST & Enhanced DSEP & IAC-BaSTI & Average \\
\hline SPZ19, BSV10, SMB18 & $0.120 \pm 0.026$ & $0.124 \pm 0.008$ & $0.135 \pm 0.009$ & $0.099 \pm 0.010$ & $0.120 \pm 0.021$ \\
SPZ19, BSV10, SMB18, Gaia, SDSS & $0.118 \pm 0.019$ & $0.126 \pm 0.008$ & $0.142 \pm 0.012$ & $0.099 \pm 0.013$ & $0.121 \pm 0.022$ \\
\hline
\end{tabular}

Fig. 16 shows the empirical extinction laws after the adjustment. It is seen that the adjustment results in a better agreement of the datasets for each model, while there is a slightly worse agreement between the different models. This residual disagreement of the models seems to reflect their inherent systematic differences, when the systematic differences between the datasets are removed.

The disagreement of the models in the $E(B-V)$ estimates in Table 3 by use of the SPZ19 dataset is \pm 0.02 mag. Similar values are obtained from the scatter of the different isochrones along $T_{\text {eff }}$ in Fig. 13: it varies from $\pm 70 \mathrm{~K}$ at the TO to $\pm 50 \mathrm{~K}$ at the RGB and MS, which corresponds to uncertainties of the $B-V$ colour of \pm 0.02 and \pm 0.015 mag, respectively (Casagrande \& VandenBerg 2014). Thus, the residual disagreement of the extinction laws given by the different models in Fig. 16 can be explained by some inherent differences between the models.

A similar residual disagreement of the models is seen in a disagreement of the $A_{\mathrm{V}}$ estimates, which change slightly after the adjustment. These estimates are presented in Table 6 as average values from the datasets of SPZ19, BSV10, and SMB18 (adopting $A_{\mathrm{y}}=A_{\mathrm{V}}$ with enough accuracy), as well as average values with the addition of the datasets of Gaia and SDSS. For them, we adopt the relations $A_{\mathrm{V}}=0.52\left(A_{\mathrm{G}_{\mathrm{BP}}}+A_{\mathrm{G}}\right)$ and $A_{\mathrm{V}}=0.48\left(A_{\mathrm{g}}+A_{\mathrm{r}}\right)$, which are close enough to any reliable extinction law. It is seen from Table 6 that both the estimates, with or without the additional datasets, give us the robust final result $A_{\mathrm{V}}=0.12 \pm 0.02$. This is the main 
result of our study. Since it is based on equation (2) or its analogues, this estimate of $A_{\mathrm{V}}$ is independent of the CCM89 or any other extinction law.

$A_{\mathrm{V}}=0.12 \pm 0.02$ and $(m-M)_{0}=14.35 \pm 0.06 \mathrm{mag}$ corresponds to an apparent $V$-band distance modulus of ( $m-$ $M)_{\mathrm{V}}=14.47 \pm 0.07 \mathrm{mag}$. This agrees with $(m-M)_{\mathrm{V}}=14.42$ mag from Harris $(1996),(m-M)_{\mathrm{F} 606 \mathrm{~W}}=14.45 \pm 0.05 \mathrm{mag}$ from VandenBerg et al. $(2013)$, and $(m-M)_{\mathrm{V}}=14.442_{-0.005}^{+0.006}$ from Wagner-Kaiser et al. (2017).

Our final consistent estimates are: $A_{\mathrm{V}}=0.12 \pm 0.02$, $E(B-V)=0.04 \pm 0.01$, and $R_{\mathrm{V}}=3.1_{-1.1}^{+1.6}$. Note that such an uncertain $R_{\mathrm{V}}$ is expected, given the uncertainties of the extinctions in Fig. 16.

The extinction laws of Schlafly et al. (2016) and CCM89 with the derived $A_{\mathrm{V}}$ and $R_{\mathrm{V}}=3.1$ are shown in Fig. 16. The curves for the laws of Fitzpatrick (1999) and Wang \& Chen (2019) would be indistinguishable from that of Schlafly et al. (2016) on the scale of Fig. 16, except for their slight deviation in the UV. All the laws agree with our results.

Similar to NGC 5904 in Paper I, we have found a rather high optical extinction for NGC 6205. It is about twice as high than the 'canonical' $A_{\mathrm{V}}=0.06 \mathrm{mag}$ [as a product of $E(B-V)=0.02$ by $R_{\mathrm{V}}=3.1$ ] from Harris (1996), in perfect agreement with the recent estimate of Wagner-Kaiser et al. (2017) mentioned in Sect. 1. As is evident from Fig. 16 and Table 3, this high extinction is due to rather high reddenings between the IR and optical bands.

\section{CONCLUSIONS}

This study generally follows Paper I in its approach to estimate distance, age and extinction law of a Galactic GC from a fitting of the isochrones to a multiband photometry. Now we consider NGC6205 (M13) instead of NGC5904 (M5) as in Paper I. We used the photometry of NGC 6205 in 34 filters from the HST, unWISE, Gaia DR2, SDSS, Pan-STARRS, SPZ19's UBVI and other datasets. These filters cover a wavelength range from about 235 to $4070 \mathrm{~nm}$, i.e. from the UV to mid-IR. Some of the photometric datasets were crossidentified with each other and with the unWISE catalogue. This allowed us to use the photometry in IR bands with nearly zero extinction as an IR extinction zero-point.

We accept the metallicity $[\mathrm{Fe} / \mathrm{H}]=-1.58$ based on the spectroscopy taken from the literature.

To fit the data, we used the following seven theoretical models of the stellar evolution: PARSEC, MIST, DSEP (the solar-scaled and $\mathrm{He}-\alpha$-enhanced), BaSTI (the solar-scaled and $\mathrm{He}-\alpha$-enhanced), and IAC-BaSTI.

From the fitting of the isochrones to the fiducial sequences we found the distance, age and reddening estimates for each pair of a colour and an isochrone in a CMD. By use of the reddening estimates we obtained empirical extinction laws, one law for each combination of a model and a dataset.

The derived empirical extinction laws, averaged over the models, agree with the laws of Fitzpatrick (1999), Schlafly et al. (2016), Wang \& Chen (2019), and CCM89 with the best-fitting $R_{\mathrm{V}}=3.1_{-1.1}^{+1.6}$.

The fitting revealed that for NGC 6205, with a dominant population enhanced both in helium and in $\alpha$ elements, the difference between solar-scaled and enhanced isochrones is quite small, and both kinds of isochrones can be used.
The CMDs, obtained with pairs of filters from different datasets but of similar effective wavelengths, show some colour offsets up to $0.04 \mathrm{mag}$ between the fiducial sequences and isochrones. We attribute these offsets to systematic differences of the datasets. However, some intrinsic systematic differences of the models/isochrones remain in our results: the derived distances and ages are systematically different for the ultraviolet, optical and infrared photometry used, as well as for the different models/isochrones. The latter is explained by the differences at a level of $\Delta T_{\text {eff }}= \pm 50 \mathrm{~K}$ [i.e. $\Delta(B-V)= \pm 0.015 \mathrm{mag}]$ between the isochrones in the $T_{\mathrm{eff}}-$ luminosity plane. This discrepancy shows that a further refinement of the theoretical models and isochrones is greatly needed.

As the main result of our study, all the empirical extinction laws, derived in the fitting of the data by PARSEC, MIST, enhanced DSEP, and IAC-BaSTI, agree with each other and with the estimates $A_{\mathrm{V}}=0.12 \pm 0.02, E(B-V)=$ $0.04 \pm 0.01 \mathrm{mag}$. These estimates perfectly agree with the recent one for NGC 6205 from Wagner-Kaiser et al. (2017), being twice as high as generally accepted. Our findings are in line with the results of Gontcharov \& Mosenkov (2017a,b, 2018), who have shown that the extinction and reddening at high Galactic latitudes behind the Galactic dust layer (the case of NGC 6205) has been underestimated by the 2D maps of SFD and Meisner \& Finkbeiner (2015), as well as by some $3 \mathrm{D}$ maps and models, which tend to provide $E(B-V) \approx 0.02$ mag for NGC 6205.

The most probable distance is $7.4 \pm 0.2 \mathrm{kpc}$. Hence, we found true distance modulus $(m-M)_{0}=14.35 \pm 0.06$ and apparent $V$-band distance modulus $(m-M)_{\mathrm{V}}=14.47 \pm 0.07$. These estimates agree with the literature estimates. The obtained parallax is $0.135 \pm 0.004$ mas, which agrees within the uncertainties with the recent estimate of the parallax from Gaia DR2 $0.109 \pm 0.04$ mas.

The age estimates vary even by use of only the optical bands: from 12.3 $\pm 0.7 \mathrm{Gyr}$ for $\mathrm{He}-\alpha-$ enhanced DSEP to $14.4 \pm$ 0.7 Gyr for MIST. The most probable age is $13.5 \pm 1$ Gyr.

Our study suggests a fruitful investigation of other Galactic GCs using the same approach.

\section{ACKNOWLEDGEMENTS}

We thank the anonymous reviewer for useful comments. We thank Santi Cassisi for providing the valuable BaSTI isochrones and his useful comments. We thank Charles Bonatto for discussion of differential reddening. We thank Frank Grundahl and Alessandro Savino for providing the Strömgren photometric results with their discussion. This research makes use of Filtergraph (Burger et al. 2013), an online data visualization tool developed at Vanderbilt University through the Vanderbilt Initiative in Data-intensive Astrophysics (VIDA) and the Frist Center for Autism and Innovation (FCAI, https://filtergraph.com). The resources of the Centre de Données astronomiques de Strasbourg, Strasbourg, France (http://cds.u-strasbg.fr), including the SIMBAD database and the X-Match service, were widely used in this study. This work has made use of BaSTI, PARSEC, MIST and DSEP web tools. This work has made use of data from the European Space Agency (ESA) mission Gaia (https://www.cosmos.esa.int/gaia), processed by 
the Gaia Data Processing and Analysis Consortium (DPAC, https://www. cosmos.esa.int/web/gaia/dpac/consortium). This study is based on observations made with the NASA/ESA Hubble Space Telescope. This publication makes use of data products from the Wide-field Infrared Survey Explorer, which is a joint project of the University of California, Los Angeles, and the Jet Propulsion Laboratory/California Institute of Technology. This work has made use of the Sloan Digital Sky Survey. This publication makes use of data products from the Pan-STARRS1 Surveys (PS1).

\section{REFERENCES}

An D. et al., 2008, ApJS, 179, 326

An D. et al., 2009, ApJ, 700, 523

Barker H., Paust N., 2018, PASP, 130, 034204 (BP18)

Bernard E. J. et al., 2014, MNRAS, 442, 2999

Bonatto C., Campos F., Kepler S. O., 2013, MNRAS, 435, 263

Brasseur C. M., Stetson P. B., VandenBerg D.A., Casagrande L., Bono G., Dall'Ora M., 2010, AJ, 140, 1672 (BSV10)

Bressan A., Marigo P., Girardi L., Salasnich B., Dal Cero C., Rubele S., Nanni A., 2012, MNRAS, 427, 127

Burger D., Stassun K. G., Pepper J., Siverd R. J., Paegert M., De Lee N. M., Robinson W. H., 2013, Astronomy and Computing, 2,40

Bustos Fierro I. H., Calderón J. H., 2019, MNRAS, 488, 3024

Cardelli J. A., Clayton G. C., Mathis J. S., 1989, ApJ, 345, 245 (CCM89)

Carretta E., Bragaglia A., Gratton R., D'Orazi V., Lucatello S., 2009, A\&A, 508, 695

Casagrande L., VandenBerg Don A., 2014, MNRAS, 444, 392

Casagrande L., VandenBerg Don A., 2018, MNRAS, 479, L102

Choi J., Dotter A., Conroy C., Cantiello M., Paxton B., Johnson B. D., 2016, ApJ, 823, 102

Choi J., Conroy C., Ting Y.-S., Cargile P. A., Dotter A., Johnson B. D., 2018, ApJ, 863, 65

Clem J. L., VandenBerg D. A., Stetson P. B., 2008, AJ, 135, 682

Cohen R. E., Moni Bidin C., Mauro F., Bonatto C., Geisler D., 2017, MNRAS, 464, 1874

Denissenkov P. A., VandenBerg D. A., Hartwick F. D. A., Herwig F., Weiss A., Paxton B., 2015, MNRAS, 448, 3314

Denissenkov P. A., VandenBerg D. A., Kopacki G., Ferguson J. W., 2017, ApJ, 849, 159

Deras D., Arellano Ferro A., Lazaro C., Bustos Fierro I. H., Calderon J. H., Muneer S., Giridhar S., 2019, MNRAS, 486, 2791

Dotter A., Chaboyer B., Jevremović D., Baron E., Ferguson J. W., Sarajedini A., Anderson J., 2007, AJ, 134, 376

Dotter A., Chaboyer B., Jevremović D., Kostov V., Baron E., Ferguson J.W., 2008, ApJS, 178, 89

Dotter A., 2016, ApJS, 222, 8

Eisenstein D. J. et al., 2006, ApJS, 167, 40

Fitzpatrick E. L., 1999, PASP, 111, 63

Gaia Collaboration et al., 2018a, A\&A, 616, A1

Gaia Collaboration et al., 2018b, A\&A, 616, A4

Goldsbury R., Richer H. B., Anderson J., Dotter A., Sarajedini A., Woodley K., 2010, AJ, 140, 1830

Gontcharov G. A., Mosenkov A. V., 2017a, MNRAS, 470, L97

Gontcharov G. A., Mosenkov A. V., 2017b, MNRAS, 472, 3805

Gontcharov G. A., Mosenkov A. V., 2018, MNRAS, 475, 1121

Gontcharov G. A., Mosenkov A. V., Khovritchev M. Yu., 2019, MNRAS, 483, 4949 (Paper I)

Grundahl F., VandenBerg D. A., Andersen M. I., 1998, ApJ, 500, L179 (GVA98)

Harris W. E., 1996, AJ, 112, 1487
Hendricks B., Stetson P. B., VandenBerg D. A., Dall'Ora M., 2012, AJ, 144, 25

Hidalgo S. L. et al., 2018, ApJ, 856, 125

Lindegren L. et al., 2018, A\&A, 616, A2

Marigo P., Bressan A., Nanni A., Girardi L., Pumo M. L., 2013, MNRAS, 434, 488

Marino A. F. et al., 2019, MNRAS, 487, 3815

Marsakov V. A., Koval' V. V., Gozha M. L., 2019, Astron. Reports, 63,274

Meisner A. M., Finkbeiner D. P., 2015, ApJ, 798, 88

Miocchi P. et al., 2013, ApJ, 774, 151

Monelli M. et al., 2013, MNRAS, 431, 2126

Nardiello D. et al., 2018, MNRAS, 481, 3382

Paltrinieri B., Ferraro F. R., Fusi Pecci F., Carretta E., 1998, MNRAS, 293, 434 (PFF98)

Paxton B., Bildsten L., Dotter A., Herwig F., Lesaffre P., Timmes F., 2011, ApJS, 192, 3

Paxton B. et al., 2013, ApJS, 208, 4

Pietrinferni A., Cassisi S., Salaris M., Castelli F., 2004, ApJ, 612, 168

Pietrinferni A., Cassisi S., Salaris M., Castelli F., 2006, ApJ, 642, 797

Pietrinferni A., Cassisi S., Salaris M., Hidalgo S., 2013, A\&A, 558, A46

Piotto G. et al., 2002, A\&A, 391, 945

Reimers D., 1975, Mem. Soc. R. Sci. Liege, 8, 369

Rey S.-C., Yoon S.-J., Lee Y.-W., Chaboyer B., Sarajedini A., 2001, AJ, 122, 3219

Rosenfield P., Marigo P., Girardi L., Dalcanton J. J., Bressan A., Williams B. F., Dolphin A., 2016, ApJ, 822, 73

Saracino S. et al., 2019, ApJ, 874, 86

Savino A., Massari D., Bragaglia A., Dalessandro E., Tolstoy E., 2018, MNRAS, 474, 4438 (SMB18)

Schlafly E. F., Finkbeiner D. P., 2011, ApJ, 737, 103

Schlafly E. F. et al., 2016, ApJ, 821, 78

Schlafly E. F., Meisner A. M., Green G. M., 2019, ApJS, 240, 30

Schlegel D. J., Finkbeiner D. P., Davis M., 1998, ApJ, 500, 525 (SFD)

Skrutskie M.F. et al., 2006, AJ, 131, 1163

Stetson P. B., 1987, PASP, 99, 191

Stetson P. B., Pancino E., Zocchi A., Sanna N., Monelli M., 2019, MNRAS, 485, 3042 (SPZ19)

VandenBerg D. A., Brogaard K., Leaman R., Casagrande L., 2013, ApJ, 775, 134

Wagner-Kaiser R. et al., 2017, MNRAS, 468, 1038

Wang S., Chen X., 2019, ApJ, 877, 116

Weiler M., 2018, A\&A, 617, A138

Welch D. L., Stetson P. B., 1993, AJ, 105, 1813

Wright E. L. et al., 2010, AJ, 140, 1868

\section{APPENDIX A: UNCERTAINTIES}

The uncertainties of the derived distance, age and reddening depend on the uncertainties of the relative positioning of the fiducial sequence and isochrone.

In addition, the uncertainty of the derived reddening includes an uncertainty of the extinction zero-point used. In turn, it is defined by an uncertainty of the extinction law used for the extinction in the reddest band of each dataset. This provides an uncertainty of the extinction zero-point at a level of several thousandths of a magnitude, as discussed in Sect. 5.1.

The most important for the fiducial sequence positioning are: 
- the photometric error from Table 1 in combination with the distribution of the stars in the CMD,

- the multiple population bias and

- the differential reddening in combination with other cluster field errors.

The most important for the isochrone positioning are:

- the uncertainty due to a wrong adopted metallicity,

- the uncertainty due to a wrong adopted He and $\alpha$ enhancement, and

- the intrinsic systematic uncertainty of the model/isochrone.

The latter can be separated into an uncertainty of each model in its application to a dataset (it seems to be reduced to a level of $<0.01 \mathrm{mag}$ by our adjustment in Sect. 6) and a residual uncertainty of each model itself. This residual uncertainty can be estimated from the model differences in Fig. 13, if we assume a similar accuracy of the models.

The following quantities are most important to derive distance, age and reddening:

(i) the mean isochrone-fiducial colour difference along the reddening vector (which is rather close to the abscissa in the CMDs) for the reddening,

(ii) the magnitudes of the HB and SGB for the distance,

(iii) the length of the SGB (the colour difference between the TO and the base of the RGB) and/or the HB-SGB magnitude difference - for the age.

For (i), to determine the fiducial sequence colour, we typically use 10-30 mag bins. For each of them the fiducial colour is determined with a precision of $0.01-0.02$ mag. Consequently, the uncertainty of the mean is between $0.01 /(30)^{0.5}=0.002$ and $0.02 /(10)^{0.5}=0.006 \mathrm{mag}$. This is the photometric error contribution to the uncertainty of the fiducial sequence colour.

The remaining uncertainties for (i) are the following. The uncertainty due to the multiple populations has been discussed in Sect. 3: typically it is $0.006 \mathrm{mag}$ and up to 0.010 mag. The uncertainty due to the differential reddening and other field errors after the cleaning of the datasets and averaging of the errors over the cluster field has been discussed in Sect. 3.1: typically it is 0.005 mag. We estimate the uncertainties due to wrong adopted metallicity and $\mathrm{He}-\alpha$-enhancement empirically, i.e. by comparison of the positions of the isochrones with different metallicity and $\mathrm{He}-\alpha$-enhancement in the same CMDs. For the former we vary $[\mathrm{Fe} / \mathrm{H}]=-1.58$ within \pm 0.04 stated by Carretta et al. (2009), while for the latter we compare the solar-scaled and enhanced isochrones from DSEP or BaSTI. It appears that each of these uncertainties varies from CMD to CMD between 0.002 and $0.012 \mathrm{mag}$, with a typical value of 0.005 mag and the maximal value for the $U-B$ versus $B$ CMD. The residual model uncertainty is at a level of $\Delta T_{\mathrm{eff}}= \pm 50$ K, i.e. $\Delta(B-V)= \pm 0.015 \mathrm{mag}$, as seen from Fig. 13 and discussed in Sect. 6.

Adding all the uncertainties, we estimate the total predicted uncertainty of the derived reddening. It is presented for each CMD in the last column of Table 3. For almost all CMDs this uncertainty is between 0.03 and 0.04 mag.

For (ii), to determine the magnitudes of the fiducial sequence's HB and SGB, we use, typically, several (e.g. six) colour bins. For each of them the magnitude is determined with a precision of a half of one magnitude bin, i.e. $0.1-0.2$ mag. Hence, the uncertainty of the mean magnitude of the fiducial sequence is between $0.1 /(6)^{0.5}=0.04$ and $0.2 /(6)^{0.5}=0.08 \mathrm{mag}$, or slightly better, if we combine the independent estimates for the SGB and HB. Figs. 2-7 show that the uncertainties due to the multiple populations and field errors have little effect on the fiducial sequence's HB and SGB. Thus, adding some insignificant multiple population bias and field error, we estimate a typical distance modulus uncertainty, due to the uncertainty of the magnitudes of the fiducial sequence's HB and SGB, as about \pm 0.1 mag. For NGC 6205 this corresponds to a typical distance uncertainty $\pm 340 \mathrm{pc}$.

The remaining uncertainties for (ii) are the following. Fig. 13 and all the CMDs show that the uncertainty of the isochrone's HB and SGB magnitude due to a wrong adopted metallicity or enhancement is negligible w.r.t. the residual intrinsic uncertainty of the models. The latter can be predicted from the scatter of the different isochrones in Fig. 13 along $L$. This scatter at the HB or SGB corresponds to a typical distance uncertainty \pm 430 or $\pm 300 \mathrm{pc}$, if the outlying enhanced BaSTI isochrone is taken into account or not, respectively. Hereafter we use the latter value, since the enhanced BaSTI is not used in the calculation of the final estimates of the distance and age. Finally, the uncertainty \pm 340 pc of the fiducial sequence's HB and SGB magnitudes dominates in the total predicted distance uncertainty.

For (iii), the photometric error contribution is the following. Typically, the length of the SGB is determined with a precision of the colours of few bins engaged at the TO and base of the RGB, i.e. $0.01-0.02 \mathrm{mag}$, and, hence, with a slightly better uncertainty of the mean. This corresponds to an age uncertainty within $0.5-1.0$ Gyr. For the HB-SGB magnitude difference we use several colour bins at the HB and SGB and, as for the case (ii), the uncertainty of the mean is typically $0.04-0.08 \mathrm{mag}$. This corresponds to an age uncertainty within $0.4-0.8$ Gyr. The contribution of the multiple populations into the age uncertainty is discussed in Sect. 3: it is within \pm 0.5 Gyr. Adding some field error, we estimate a typical age uncertainty due to the uncertainty of the fiducial sequence, as about \pm 1 Gyr.

For (iii), similarly to (ii), Fig. 13 and all the CMDs show that the uncertainty of the derived age due to the uncertainty of the isochrone is dominated by the residual intrinsic uncertainty of the models. A typical uncertainty of the model HB-SGB magnitude difference and the length of the SGB can roughly be predicted from Fig. 13 as corresponding to an age uncertainty about \pm 1 Gyr. However, an additional uncertainty of the SGB length may be added due to some uncertainties of the colour- $T_{\text {eff }}$ relations and bolometric corrections used, which are not taken into account in Fig. 13.

In some cases the length of the SGB and the HB-SGB magnitude difference provide contradictory results. For example, in Fig. 9 (c) the IAC-BaSTI isochrone of 14.5 Gyr is a compromise between too long an SGB and too big an HBSGB magnitude difference: a shorter observed SGB better fits an age about 15 Gyr, while a shorter observed HB-SGB difference better fits an age about 14 Gyr. In such a case, our search for a minimal total offset between the isochrone and fiducial points provides a compromise solution. 

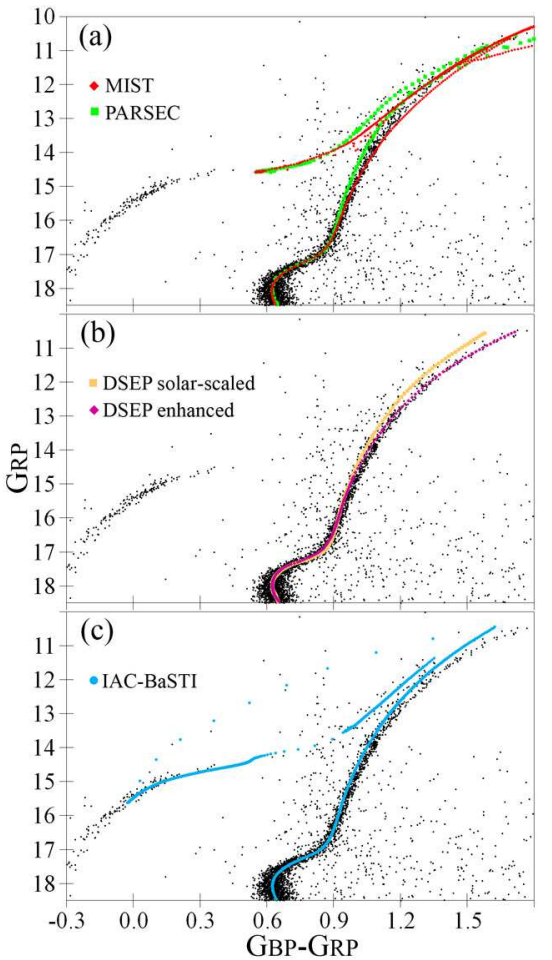

Figure B1. The same as Fig. 9 but for Gaia DR2 $G_{\mathrm{BP}}-G_{\mathrm{RP}}$ versus $G_{\mathrm{RP}}$.

Finally, this analysis of the predicted uncertainties shows that the intrinsic systematic uncertainty of the models and isochrones appears to be one of the main contributions into the total predicted uncertainty. This shows a need for a further refinement of the models and isochrones.

\section{APPENDIX B: SOME CMDS OF NGC 6205}

This paper has been typeset from a $\mathrm{T}_{\mathrm{E}} \mathrm{X} / \mathrm{LAT} \mathrm{EX}$ file prepared by the author.
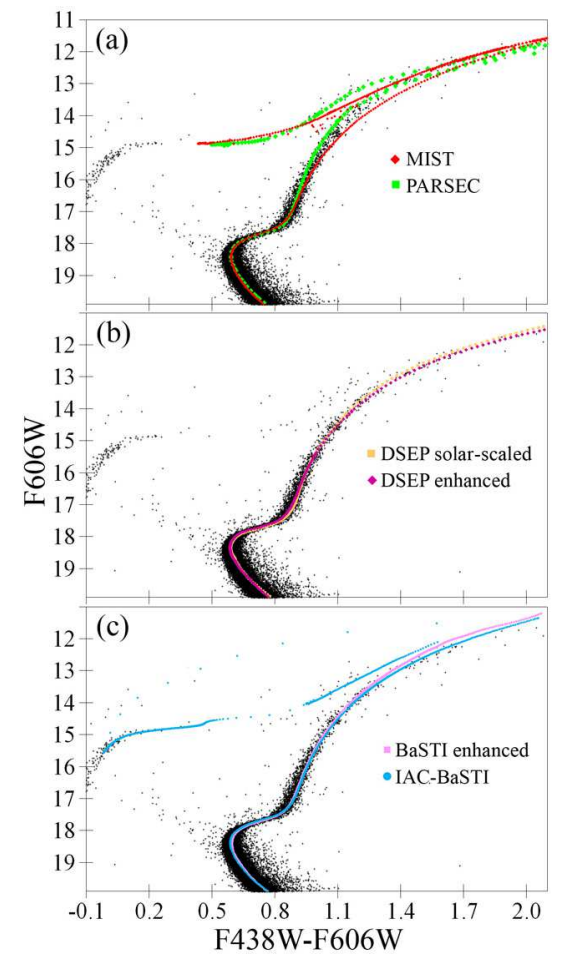

Figure B2. The same as Fig. 9 but for HST ACS/WFC3 $F 438 W-F 606 W$ versus $F 606 W$ based on the data from Nardiello et al. (2018).
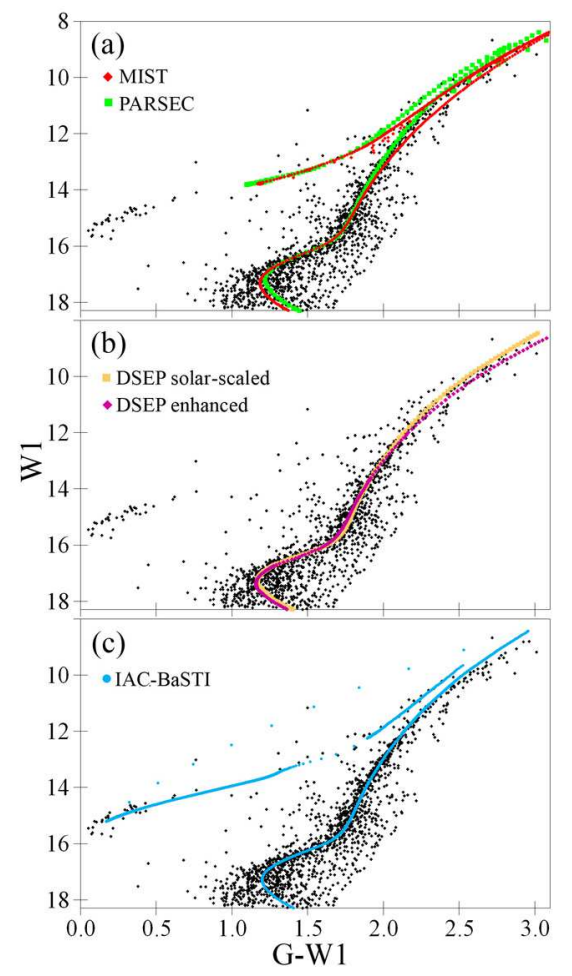

Figure B3. The same as Fig. 9 but for $G-W 1$ versus $W 1$ based on the data from Gaia DR2 and unWISE. 


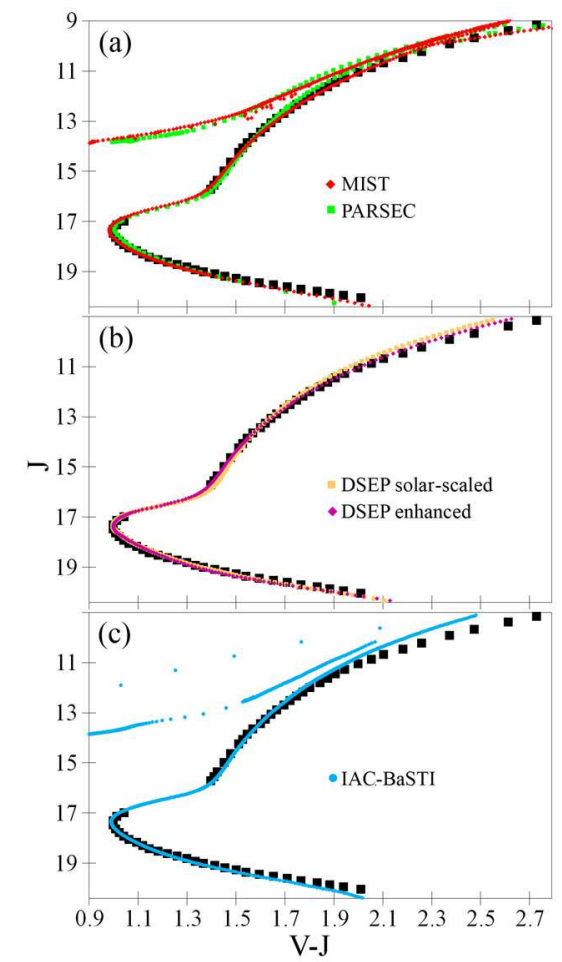

Figure B4. $\boldsymbol{V}-\boldsymbol{J}$ versus $\boldsymbol{J}$ CMD of NGC 6205 with the fiducial sequence from BSV10 (black squares), and the isochrones calculated with the best-fitting parameters from Table 3 and coloured as in Fig. 9. 